\title{
Heat transfer and hybrid nanofluid flow over a porous stretching/shrinking sheet with Brinkmann model and multiple slips
}

Thongchai Botmart ( $\sim$ thongbo@kku.ac.th )

Khon Kaen University

\section{U. S. Mahabaleshwar}

Davangere University

\section{A. B. Vishalakshi}

Davangere University

\section{Research Article}

Keywords: Brinkmann number, porous medium, stability analysis, hybrid nanofluid, Kummer's function, velocity and temperature profile

Posted Date: March 1st, 2022

DOI: https://doi.org/10.21203/rs.3.rs-1385643/v1

License: (a) (1) This work is licensed under a Creative Commons Attribution 4.0 International License. Read Full License 


\title{
Heat transfer and hybrid nanofluid flow over a porous stretching/shrinking sheet with Brinkmann model and multiple slips
}

\author{
U. S. Mahabaleshwar ${ }^{1}$, A.B. Vishalakshi ${ }^{1}$ and Thongchai Botmart ${ }^{2 *}$ \\ ${ }^{1}$ Department of Mathematics, Davangere University, Shivagangothri, Davangere, 577 007, \\ INDIA, Email: u.s.m@davangereuniversity.ac.in, vishalavishu691@gmail.com \\ ${ }^{2}$ Department of Mathematics, Faculty of Science, Khon Kaen University, Khon Kaen 40002, \\ Thailand \\ * Corresponding author: thongbo@kku.ac.th
}

\begin{abstract}
The current study explains the hybrid nanofluid flow and transfer of heat with radiation and mass transpiration. The velocity slips and thermal jump condition is also taken in governing equation respectively. The solid volume fractions of $\mathrm{Al}_{2} \mathrm{O}_{3}-\mathrm{Cu} / \mathrm{H}_{2} \mathrm{O}$ hybrid nanofluid are considered tin the entire calculation. The governing PDEs are altered into ODEs with the help of similarity variable. Hybrid nanofluid is used to increase the efficiency of heat transfer and in the field of industrial applications. The role of Brinkmann model is considered in the momentum equations. Momentum equation is solved analytically to get the $4^{\text {th }}$ root of the equation and also get the solution domain. This domain is used in the energy equation and then it is solved analytically and expressed in terms of Kummer's function. The existence and nonexistence solutions for both stretching and shrinking sheet is investigated. Shrinking sheet case exhibit various behavior compared to stretching sheet case and produces physically meaningful dual solutions. The results of velocity and temperature profiles can be analyzed with the help of different controlling parameters namely mass transpiration, thermal radiation, solid volume fractions, Brinkmann number, and porous medium parameter. Stability analysis can be performed to extend the results of the present study.
\end{abstract}

Key words: Brinkmann number, porous medium, stability analysis, hybrid nanofluid, Kummer's function, velocity and temperature profile. 
NOMENCLATURE

List of variables

$A \& B$

$a$

$b_{1} \& b_{2}$

$c_{1}$

$C_{P}$

$d$

$f$

$f_{\eta}$

F

$k^{*}$

K

$\operatorname{Pr}$

$Q_{0}$

$N i$

$q_{r}$

$R$

$T_{\infty}$

$T_{w}$
Description

S.I. Units

Unknowns to be determined

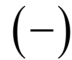

Constant

$(-)$

Coefficients of velocity slips

$(-)$

Temperature jump constant

Specific heat at constant pressure

$\left(J k g^{-1} K^{-1}\right)$

Stretching/shrinking parameter $\left(=\frac{\alpha_{1}}{a}\right)$

Non dimensional transverse velocity

Non dimensional Tangential velocity

Kummer's function

Mean absorption coefficient

The permeability of porous medium

$\left(m^{2}\right)$

Prandtl number

Coefficient of Heat source/sink parameter

Heat source/sink parameter $\left(=\frac{Q_{0}}{\rho C_{P} a}\right)$

Radiative heat flux.

Radiation parameter $\left(=\frac{16 \sigma^{*} T_{\infty}^{3}}{3 k^{*} \kappa_{f}}\right)$,

Temperature at free stream

Wall temperature 
$(u, v)$

$u_{w}(x)$

$v_{w}$

$V_{C}$

\section{Greek symbols}

$\alpha$

$\beta$

$\delta$

$\phi_{1} \& \phi_{2}$

$\lambda$

$\xi$

$\Lambda$

$\varepsilon$

v

$\mu$

$\kappa$

$\theta$
Components of velocities

$\left(m s^{-1}\right)$

$\left(m s^{-1}\right)$

Mass transfer velocity

$\left(m s^{-1}\right)$

Mass transpiration $\left(=-\frac{v_{w}}{\sqrt{a v_{f}}}\right)$

$(-)$

Temperature jump parameter $\left(=c_{1} \sqrt{\frac{a}{v_{f}}}\right)$

Kinematic viscosity

$\left(m^{2} s^{-1}\right)$

Dynamic viscosity

$\left(\mathrm{kgm}^{-1} \mathrm{~s}^{-1}\right)$

Thermal conductivity

$\left(W m^{-1} K^{-1}\right)$

Non-dimensional temperature 


$\begin{array}{llr}\sigma^{*} & \text { Stefan-Boltzmann constant } & \left(\mathrm{Wm}^{-2} \mathrm{~K}^{-1}\right) \\ \rho & \text { Density of the fluid } & \left(\mathrm{kgm}^{-3}\right) \\ \psi(x, y) & \text { Stream function } & \left(\mathrm{kgm}^{-1} \mathrm{~s}^{-1}\right)\end{array}$

\section{Subscripts}

$\begin{array}{ll}w & \text { Wall condition } \\ \infty & \text { Free stream condition } \\ \eta & \text { Differentiation with respect to } \eta \\ s_{1} \& s_{2} & \text { First and second fluid solid nanoparticle respectively } \\ f & \text { Base fluid } \\ h n f & \text { Hybrid nanofluid }\end{array}$

\section{Abbreviations}

B. Cs

Boundary conditions

ODE

Ordinary differential equations

PDE

Partial differential equations

\section{Introduction:}

Nowadays enhancement of heat transfer plays a major role in the industrial applications such as biomedical, electronics, transportation applications namely auto-mobiles, nuclear system power engine. Many innovative methods have been conducted to increase the enhancement of heat transfer [1]. Maxwell [2] discovered that enhancement of heat transfer is possible by immersed some substances into the fluid. Choi and Eastman [3] depicted that theory of nanofluid is a finest form of heat transfer. Nanofluid is the nanoparticles and base fluids colloidal mixture. Aly[4] worked on dual solution of graphene water nanofluid with magnetic field, from this he concluded that grhene nanoparticles act as a cooler when we increases the magnetic field effect. Later Benos et al. [5] investigated the asymptotic analysis of MHD 
natural convection nanofluid with porous enclosure. Recently Mahabaleshwar et al. [6-7] worked on nanofluids for flow problems with role of Brinkmann model and thermal radiation under variety of boundary conditions and different medium namely porous medium. The fluid flow due to porous sheet containing numerous applications, the different equations are found to explain the flow of a fluid due to porous sheet. Mahabaleshwar et al. [8-11] worked on the effect of Brinkman model, role of Navier's slip on stretching sheet problems under the effect of thermal radiation and mass transpiration with porous medium. Tamayol et al. [12] describe the exact solutions of boundary layer flow due to a porous sheet. Anusha et al. [13] describe the Navier's slip effect under porous sheet with mass transfer.

In recent investigations hybrid nanofluid is utilized as the advancement of nanofluid, because compare to base fluid and nanofluid hybrid nanofluid exhibit better thermal efficiency. Sarkar et al. [14] worked on developments of hybrid nanofluid and he conclude that a thermal efficiency is affected by proper technique of hybridization. Later Anusha et al. [15-16] and Sneha et al. [17] worked on hybrid nanofluid flow with thermal radiation and mass transpiration, from this studies it is conclude that the hybrid nanofluid enhances the thermal efficiency of the fluid. see some other references of hybrid nanofluid given in ref. [18-19].

On the proper knowledge of all the above references the present work is conducted an experiment on hybrid nanofluid flow over a porous sheet with thermal radiation and mass transpiration. $\mathrm{Al}_{2} \mathrm{O}_{3}-\mathrm{Cu} / \mathrm{H}_{2} \mathrm{O}$ hybrid nanofluid is used to analyze the present study. The effect of Brinkmann model and mass transpiration is considered in the momentum equation also the effect of thermal radiation and heat source/sink parameter is considered under energy equation. Then the momentum equation is solved analytically to get the $4^{\text {th }}$ root of equation, after solving this equation to get the solution domain. This domain is used in the energy equation, this equation solved analytically and expressed in terms of Kummer's function. The present problem is well argument with Usafzai et al. [20]. 


\section{Problem statement and solution}

Flow of 2-D flow with heat transfer under the impact of thermal radiation, Brinkmann model and mass transpiration is investigated in the present analysis. The solid volume fractions of $\mathrm{Al}_{2} \mathrm{O}_{3}-\mathrm{Cu} / \mathrm{H}_{2} \mathrm{O}$ nanoparticles are considered in the entire calculation. Schematically the fluid flow is represented at Fig.1. and also the quantities of hybrid nanofluids are indicated at Table.1. A steady laminar flow over a porous sheet is placed at $y>0, x$-axis moves along the sheet and y-axis is placed normal to it. Let $T_{\infty}$ is assumed to be ambient temperature from the for field. The fluid is maintained at the constant surface temperature $T_{w}$. Then the fluid flow phenomenon is modelled into the following form of governing equation are as follows

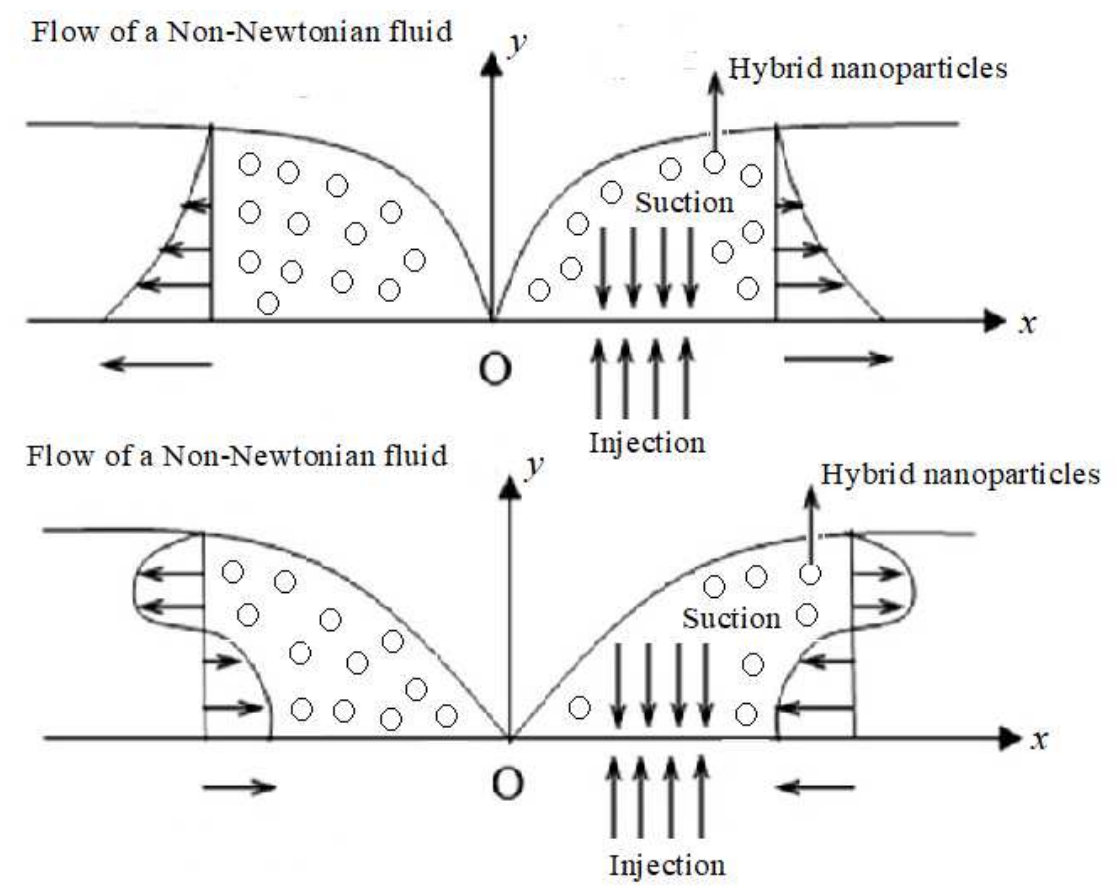

Fig.1: The schematic diagram of a non Newtonian flow

$\frac{\partial u}{\partial x}+\frac{\partial v}{\partial y}=0$

$u \frac{\partial u}{\partial x}+v \frac{\partial u}{\partial y}=\frac{\mu_{e f f}}{\rho_{h n f}}\left(1+\frac{1}{\lambda}\right) \frac{\partial^{2} u}{\partial y^{2}}-\frac{\mu_{h n f}}{\rho_{h n f} K} u$

$u \frac{\partial T}{\partial x}+v \frac{\partial T}{\partial y}=\frac{\kappa_{h n f}}{\left(\rho C_{P}\right)_{h n f}} \frac{\partial^{2} T}{\partial y^{2}}-\frac{1}{\left(\rho C_{P}\right)_{h n f}} \frac{\partial q_{r}}{\partial y}+\frac{Q_{0}\left(T-T_{\infty}\right)}{\left(\rho C_{P}\right)_{h n f}}$, 
B. Cs associated with these modelled equations are given by

$$
\begin{aligned}
& u(x, \infty)=0, \quad v(x, 0)=v_{w} \\
& u(x, 0)=u_{w}(x)+b_{1} \frac{\partial u}{\partial y}+b_{2} \frac{\partial^{2} u}{\partial y^{2}}, \\
& T(x, \infty)=T_{\infty}, \quad T(x, 0)=T_{w}(x)+c_{1} \frac{\partial T}{\partial y}
\end{aligned}
$$

The terms used in Eq. (1) to (4) are defined in the Nomenclature

To transfer the fluid flow and temperature flow we use the following similarity variables in terms of stream function as follows

$$
\psi(x, y)=\sqrt{a v_{f}} f(\eta), \quad \eta=y \sqrt{\frac{a}{v_{f}}},
$$

by using stream function, the velocities can be defined as

$$
u=\operatorname{axf}_{\eta}(\eta), \quad v=-\sqrt{a v_{f}} f(\eta) .
$$

Radiation effect is considered in the energy equation, it can be calculated by using Rosseland's approximation, on the basis of this formula $q_{r}$ can be defined as follows

$$
q_{r}=-\frac{4 \sigma^{*}}{3 k^{*}} \frac{\partial T^{4}}{\partial y},
$$

The terms used in Eq. (7) are defined in the Nomenclature.

With the help of Taylor's series $T^{4}$ can be expanded and then ignoring some terms to get the equation as

$$
T^{4} \cong-3 T_{\infty}^{4}+4 T_{\infty}^{3} T
$$

Upon using equation above equations one can obtain (see Ref. [21-24])

$$
\frac{\partial q_{r}}{\partial y}=-\frac{16 \sigma^{*} T_{\infty}^{3}}{3 k^{*}} \frac{\partial^{2} T}{\partial y^{2}}
$$

With the help of similarity variables defined in Eq. (5) and (6), also using Eq. (9) in governing equations (1) to (3) to get the following ODEs (see Ref. [25]) 


$$
\begin{aligned}
& \Lambda\left(1+\frac{1}{\lambda}\right) f_{\eta \eta \eta}+r_{2}\left(f f_{\eta \eta}-f_{\eta}^{2}\right)-r_{1} \varepsilon f_{\eta}=0, \\
& \frac{1}{\operatorname{Pr}}\left(r_{4}+R\right) \theta_{\eta \eta}+r_{3}\left(f \theta_{\eta}-f_{\eta} \theta\right)+N i \theta=0,
\end{aligned}
$$

the B. Cs defined in Eq. (4) also reduced to

$$
\left.\begin{array}{l}
f_{\eta}(\infty)=0, \quad f(0)=V_{C} \\
f_{\eta}(0)=d+\alpha f_{\eta \eta}(0)+\beta f_{\eta \eta \eta}(0) \\
\theta(\infty)=0, \gamma \theta_{\eta}(0)-\theta(0)+1=0
\end{array}\right\} .
$$

Here, $\Lambda=\frac{\mu_{e f f}}{\mu_{f}}, \quad \varepsilon=\frac{v_{f}}{a K}, N i=\frac{Q_{0}}{\rho C_{P} a}$, and $R=\frac{16 \sigma^{*} T_{\infty}^{3}}{3 k^{*} \kappa_{f}}$, represents Brinkmann number, porous medium parameter, heat source/sink parameter and thermal radiation respectively. Mass transpiration $V_{C}=-\frac{v_{w}}{\sqrt{a v_{f}}}$, if $V_{C}>0$ indicates suction and if $V_{C}<0$ indicates injection at the wall. The quantity $d=\frac{\alpha_{1}}{a}$ indicates stretching/shrinking sheet parameter. Additionally, in the above system $\alpha=b_{1} \sqrt{\frac{a}{v_{f}}}, \beta=b_{2}\left(\frac{a}{v_{f}}\right)$, and $\gamma=c_{1} \sqrt{\frac{a}{v_{f}}}$ indicates first and second order slips and temperature jump respectively.

The quantities of hybrid nanofluid used in the Eq. (10) and (11) can be defined as follows

$$
\begin{aligned}
& r_{1}=\frac{\mu_{h n f}}{\mu_{f}}=\frac{1}{\left(1-\phi_{1}\right)^{2.5}\left(1-\phi_{2}\right)^{2.5}} \\
& r_{2}=\frac{\rho_{h n f}}{\rho_{f}}=\left(1-\phi_{2}\right)\left(1-\phi_{1}+\phi_{1} \frac{\rho_{S_{1}}}{\rho_{f}}\right)+\phi_{2} \frac{\rho_{S_{2}}}{\rho_{f}} \\
& r_{3}=\frac{\left(\rho C_{P}\right)_{h n f}}{\rho_{f}}=\left(1-\phi_{2}\right)\left(1-\phi_{1}+\phi_{1} \frac{\left(\rho C_{P}\right)_{S_{1}}}{\left(\rho C_{P}\right)_{f}}\right)+\phi_{2} \frac{\left(\rho C_{P}\right)_{S_{2}}}{\left(\rho C_{P}\right)_{f}} \\
& r_{4}=\frac{\kappa_{h n f}}{\kappa_{f}}=\frac{\kappa_{S_{2}}+\kappa_{b f}+2 \phi_{2}\left(\kappa_{S_{2}}-\kappa_{f}\right)}{\kappa_{S_{2}}+\kappa_{b f}-\phi_{2}\left(\kappa_{S_{2}}-\kappa_{f}\right)} \\
& \text { where, } \frac{\kappa_{h n f}}{\kappa_{f}}=\frac{\kappa_{S_{1}}+\kappa_{b f}+2 \phi_{1}\left(\kappa_{1}-\kappa_{f}\right)}{\kappa_{S_{1}}+\kappa_{b f}-\phi_{1}\left(\kappa_{S_{1}}-\kappa_{f}\right)}
\end{aligned}
$$


Table.1: Thermophysical properties of hybrid nanofluids

\begin{tabular}{|c|c|c|c|c|}
\hline S. R. No & Thermo Physical properties & Base fluid & $\left(\mathrm{Al}_{2} \mathrm{O}_{3}\right)-\phi_{1}$ & $(\mathrm{Cu})-\phi_{2}$ \\
\hline 1 & $\rho\left(\mathrm{kg} / \mathrm{m}^{3}\right)$ & 997.1 & 3970 & 8933 \\
\hline 2 & $C_{p}(\mathrm{~J} / \mathrm{kg} \mathrm{K})$ & 4179 & 765 & 385 \\
\hline 3 & $\kappa(W / m K)$ & 0.613 & 40 & 401 \\
\hline
\end{tabular}

\section{Analytical solutions of momentum}

On the basis of physically meaningful solution of the parameters. Let us consider the solution of Eq. (10) is of the form

$f(\eta)=A+B e^{-\delta \eta}$

With the help of Eq. (4) in Eq. (14) to obtain the following results

$V_{C}=A+B$

$A=V_{C}+\frac{d}{\delta\left(1+\alpha \delta-\beta \delta^{2}\right)}, B=\frac{-d}{\delta\left(1+\alpha \delta-\beta \delta^{2}\right)}$

Using Eq. (15) and (16) in Eq. (10) to get the following $4^{\text {th }}$ root of the equation

$$
\begin{array}{r}
\Lambda\left(1+\frac{1}{\lambda}\right) \beta \delta^{4}-\left(\Lambda \alpha+r_{2} V_{C} \beta\right) \delta^{3}-\left(\Lambda-r_{2} V_{C} \alpha+r_{1} \varepsilon \beta\right) \delta^{2}+ \\
\left(r_{2} V_{C}+r_{1} \varepsilon \alpha\right) \delta+\left(r_{2} d+r_{1} \varepsilon\right)=0
\end{array},
$$

Also, Eq. (14) can be rewritten as

$$
f(\eta)=V_{C}+\frac{d\left(1-e^{-\delta \eta}\right)}{\delta\left(1+\alpha \delta-\beta \delta^{2}\right)},
$$

Eq. (18) satisfy the momentum equation (10), and for physically meaningful flow $\delta>0$.

Then the velocity and wall shear stress is obtained from Eq. (18) as

$$
f_{\eta}(\eta)=\frac{d e^{-\delta \eta}}{1+\alpha \delta-\beta \delta^{2}}
$$


$f_{\eta \eta}(0)=-\frac{d \delta}{1+\alpha \delta-\beta \delta^{2}}$

Eq. (17) and Eq. (18) work gives well argument with many research articles. If $d=r_{1}=r_{2}=1, \Lambda=\beta=0$ in Eq. (17) and Eq. (18) it reduces to Tamayol et al. [12], Crane [26] solution is recovered if $d=r_{1}=r_{2}=1, \Lambda=\alpha=V_{C}=\beta=0$. For shrinking case $d=r_{1}=r_{2}=1, \Lambda=\beta=\varepsilon=0$ the solution turns to Miklavcic and Wang [27]. If $d=r_{1}=r_{2}=1, \Lambda=\beta=0, \varepsilon>0$, the solution reduces to Fang and Zhang [28]. If $\phi_{1}>0, \phi_{2}=\Lambda=\beta=0$, the solution reduces to Usafzai et al. [20].

\section{Analytical solution for energy equation}

In this section we introduce a variable as

$\xi=-\operatorname{Exp}(-\delta \eta)$

By using Eq. (21) into Eq. (11) to get the following resulting equation

$$
\xi \theta_{\eta \eta}(\xi)+(p-q \xi) \theta_{\eta}(\xi)+\left(q+\frac{\omega}{\delta^{2} \xi}\right) \theta=0,
$$

B. Cs related with Eq. (22) is also reduces to

$$
\theta(0)=0, \delta \gamma \theta_{\eta}(-1)+\theta(-1)-1=0
$$

The dummy variables used in Eq. (22) is given by

$$
\begin{aligned}
& p=1-\frac{\Omega\left(d+V_{C} \delta\left(1+\alpha \delta-\beta \delta^{2}\right)\right)}{\delta^{2}\left(1+\alpha \delta-\beta \delta^{2}\right)} \\
& q=\frac{\Omega d}{\delta^{2}\left(1+\alpha \delta-\beta \delta^{2}\right)}, \\
& \text { here, } \Omega=\frac{\operatorname{Pr} r_{3}}{r_{4}+R}, \quad \omega=\frac{N i \operatorname{Pr}}{r_{4}+R}
\end{aligned}
$$

Then the solution of Eq. (22) after the backward substitution of $\eta$ reads as

$$
\theta(\eta)=\frac{\left(-e^{-\delta \eta}\right) F\left[m-1,1+t,-q e^{-\delta \eta}\right]}{\frac{(m-1) q \delta \gamma}{(1+t)} F[m, 2+t,-q]-(1+\gamma m \delta) F[m-1,1+t,-q]},
$$


here,

$$
m=\frac{(1-p)+\sqrt{(1-p)^{2}-\frac{4 \omega}{\delta^{2}}}}{2}, \quad t=\frac{\sqrt{(1-p)^{2}-\frac{4 \omega}{\delta^{2}}}}{2} .
$$

Where, $F[a, b, z]$ indicates Kummer's function. Next we discuss the discussion of the present work with graphical scenario.

\section{Results and discussion}

A 2-D hybrid nanofluid flow due to porous sheet with mass transpiration and radiation, source/sink parameter is investigated in the current article. The PDEs of the governing equations are altered into ODEs by using suitable transformations. Then the energy and momentum equations solved analytically with the help of various controlling parameters. The flow of the solution depends on stretching/shrinking parameter, mass transpiration, Brinkmann number, solid volume fraction, porous medium parameter, and slip parameter.

Fig.2 depict that the impact of solution domain $\delta$ on $V_{C}$ for various choices of $\varepsilon$. Fig.2a represents stretching case and Fig.2b depicts shrinking case, The physically meaningful are obtained for both $V_{C}>0$ and $V_{C}<0$. In Fig.2a it is observed that the domain $\delta_{1}$ and $\delta_{2}$ values increases with increases of the values of $\varepsilon$. This case is reversed at the domains $\delta_{3}$ and $\delta_{4}$. i.e. domain values decreases with increases of the values of $\varepsilon$. Similarly, in Fig.2b it is observed that domain $\delta_{1}$ and $\delta_{3}$ values increases with increases of the values of $\varepsilon$. This case is reversed at the domains $\delta_{2}$ and $\delta_{4}$. i.e. domain values decreases with increases of the values of $\varepsilon$. Fig.3a and Fig.3b indicates the impact of solution domain $\delta$ on $V_{C}$ for taking the single values of all the parameters at stretching and shrinking cases respectively, these graphs are drawn for taking all the four roots $\delta_{1}, \delta_{2} \delta_{3}$ and $\delta_{4}$. Fig.4a and $4 \mathrm{~b}$ indicates the effect of solution domain $\delta$ on $\varepsilon$, by taking single values of all the other parameters. Fig.4a represents stretching case and Fig.4b represents shrinking case. 
Fig.5a and Fig.5b indicates that the effect of $f_{\eta}(\eta)$ and $f_{\eta \eta}(\eta)$ on $\eta$ for various choices of $\alpha$. Fig,5a depicts the stretching sheet case, in this it is observed that the physically meaningful solution of $f_{\eta}(\eta)$ is occurred in positive $y$-axis, also $f_{\eta \eta}(\eta)$ is occurred in negative y-axis, here $f_{\eta}(\eta)$ is more for less values of $\alpha$. And $f_{\eta \eta}(\eta)$ is more for more values of $\alpha$. Fig,5b depicts the shrinking sheet case, in this it is observed that the physically meaningful solution of $f_{\eta}(\eta)$ is occurred in negative $y$-axis, also $f_{\eta \eta}(\eta)$ is occurred in positive $y$-axis, here $f_{\eta \eta}(\eta)$ decreases with increases the values of $\alpha$. And $f_{\eta}(\eta)$ increases with increase the values of $\alpha$ Fig.6a and Fig.6b indicates that the effect of $f_{\eta}(\eta)$ and $f_{\eta \eta}(\eta)$ on $\eta$ for various choices of $\alpha$ for suction and injection cases respectively. Fig.6a depicts the suction case, in this it is observed that the physically meaningful solution of $f_{\eta}(\eta)$ is occurred in positive $y$-axis, also $f_{\eta \eta}(\eta)$ is occurred in negative $y$-axis. In this $f_{\eta}(\eta)$ decreases with increase the values of $\alpha$. And $f_{\eta \eta}(\eta)$ increases with increases the values of $\alpha$. Fig.6b depicts the shrinking sheet case, in this it is observed that the physically meaningful solution of $f_{\eta}(\eta)$ is occurred in negative $y$-axis, also $f_{\eta \eta}(\eta)$ is occurred in positive $y$-axis. Here, $f_{\eta \eta}(\eta)$ decreases with increase the values $\alpha$. And $f_{\eta}(\eta)$ is more for more values of $\alpha$.

Fig.7a and Fig.7b indicates that the effect of $f_{\eta}(\eta)$ and $f_{\eta \eta}(\eta)$ on $\eta$ for various choices of $\varepsilon$ for $d>0$ and $d<0$ respectively. Fig.7a depicts the stretching case, in this it is observed that the physically meaningful solution of $f_{\eta}(\eta)$ is occurred in negative $y$-axis, also $f_{\eta \eta}(\eta)$ is occurred in positive $y$-axis. Here, $f_{\eta}(\eta)$ is more for more values of $\varepsilon$, and $f_{\eta \eta}(\eta)$ decreases with increases the values of $\varepsilon$. Fig.7b depicts the shrinking case, in this it is observed that the physically meaningful solution of $f_{\eta}(\eta)$ is occurred in positive $y$-axis, also $f_{\eta \eta}(\eta)$ is occurred 
in negative $y$-axis. Here, $f_{\eta}(\eta)$ decreases with increase the values of $\varepsilon$, and $f_{\eta \eta}(\eta)$ increases with increases the values of $\varepsilon$.

Fig.8-10 demonstrate that the impact of $\theta(\eta)$ on $\eta$ for various values of different physical parameters. Fig.8a and Fig.8b indicates that the impact of $\theta(\eta)$ on $\eta$ for various choices of $V_{C}$ for $d>0$ and $d<0$ respectively, in both the cases the $\theta(\eta)$ decreases with increases the values of $V_{C}$, also it is observed that the thickness of the boundary layer flow is more for shrinking case compare to stretching case. Fig.9a and Fig.9b indicates that the impact of $\theta(\eta)$ on $\eta$ for different values of $\gamma$ for $d>0$ and $d<0$ respectively, in both the cases the $\theta(\eta)$ is less for more values of $\gamma$. Fig.10a and Fig.10b represents the effect of $\theta(\eta)$ on $\eta$ for various choices of $\alpha$, here it is seen that $\theta(\eta)$ increases with increases the values of $\alpha$ for stretching case, reverse impact is observed at shrinking case i.e. $\theta(\eta)$ decreases with increases of $\alpha$ for shrinking case. The pattern of streamline flows is observed at Fig.11a and Fig.11b. for $V_{C}>0$ and $V_{C}<0$ cases respectively, we observe the different line patterns at $V_{C}>0$ and $V_{C}<0$. 


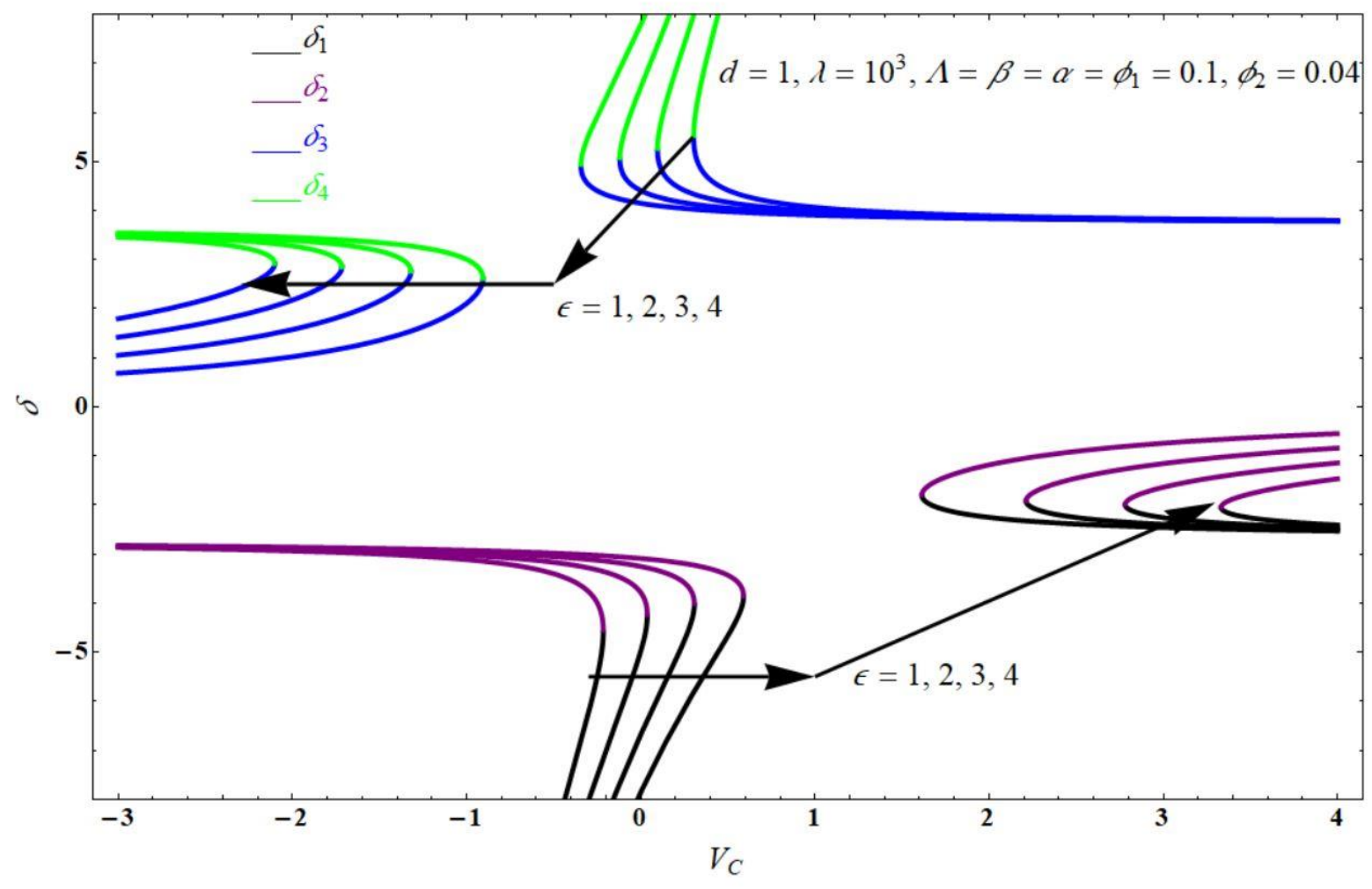

(a)

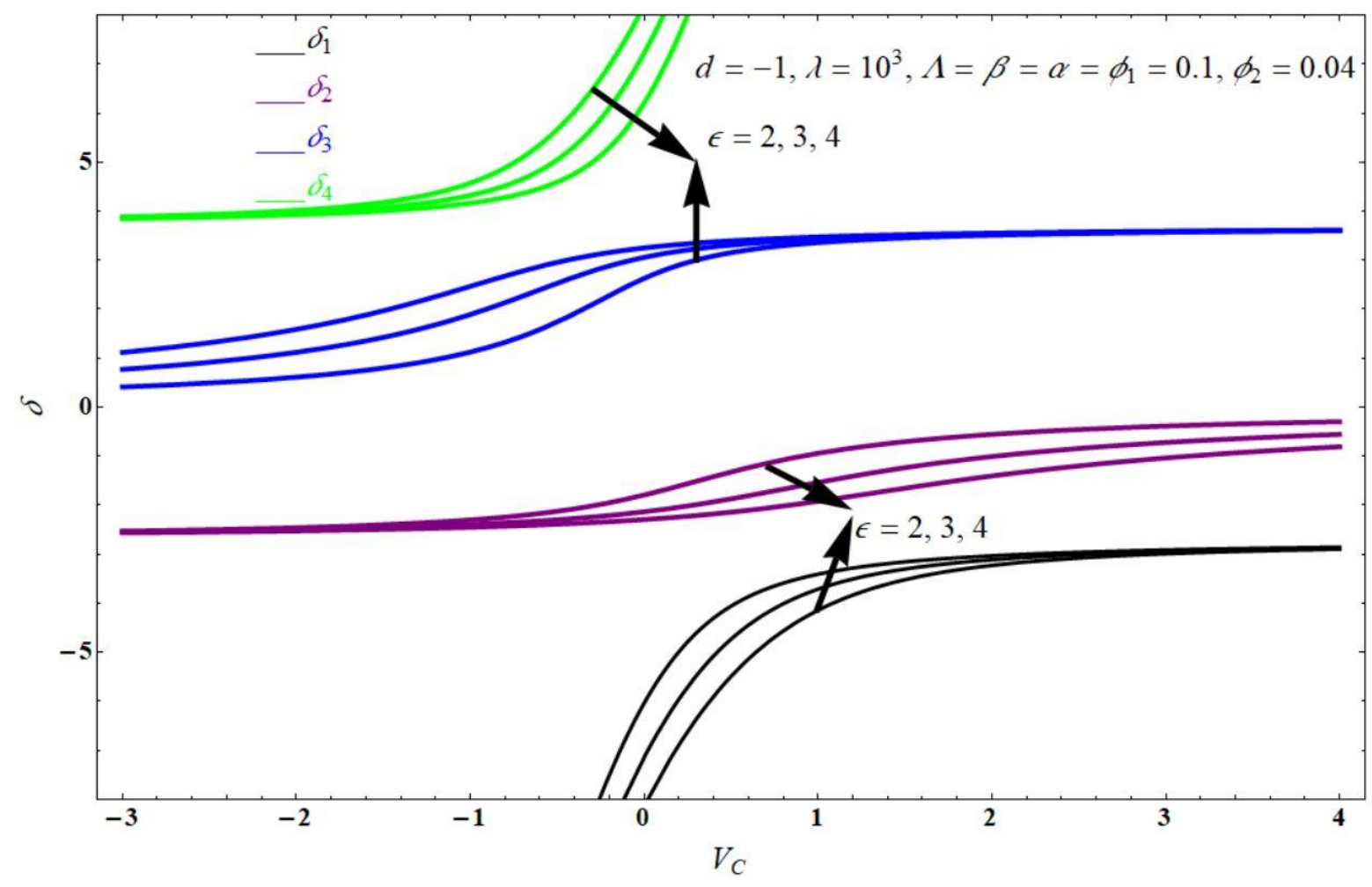

(b)

Fig.2: Impact of $\delta$ on $V_{C}$ for different choices of $\varepsilon$ at (a) $d>0$ and (b) $d<0$. 


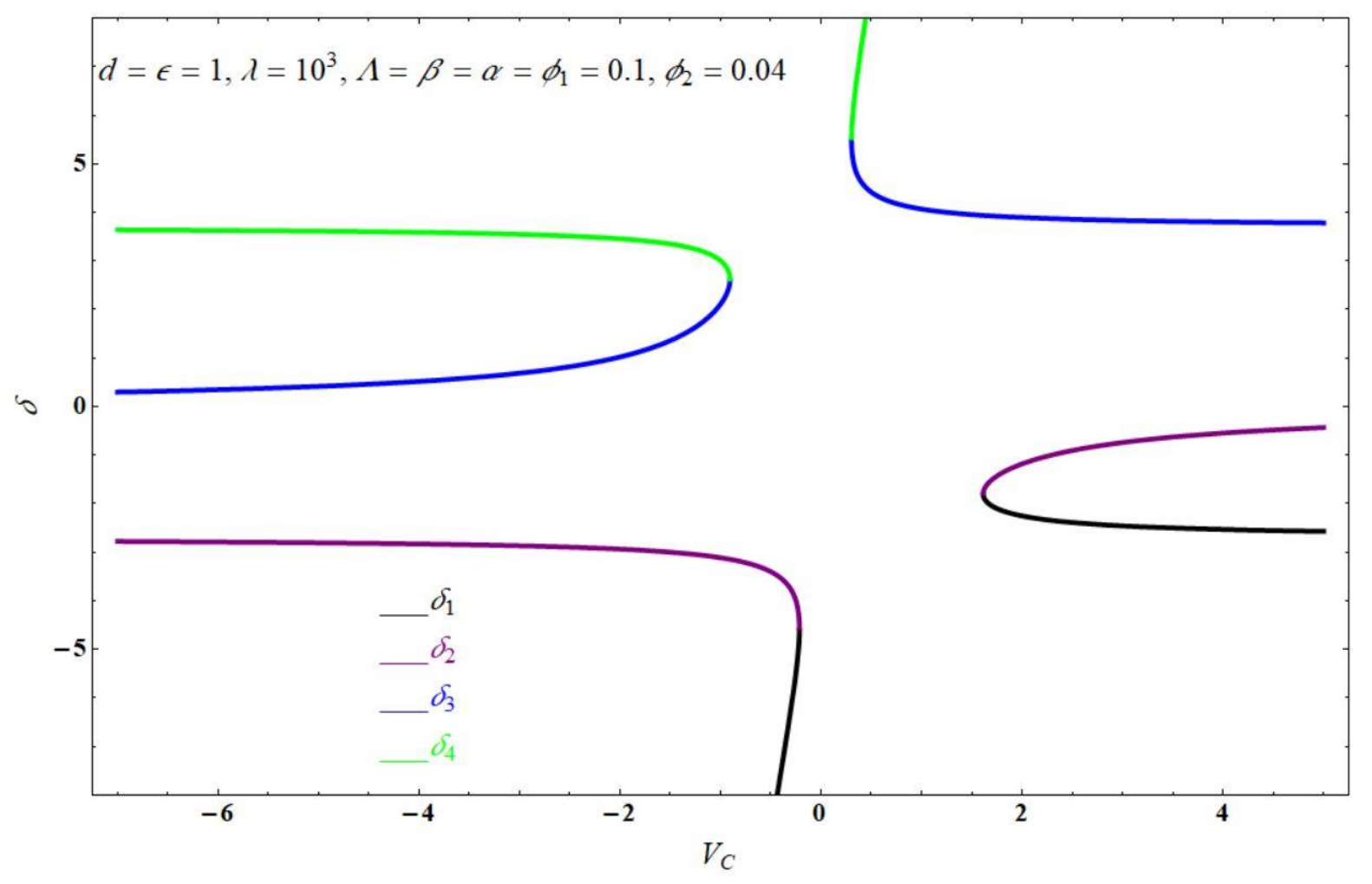

(a)

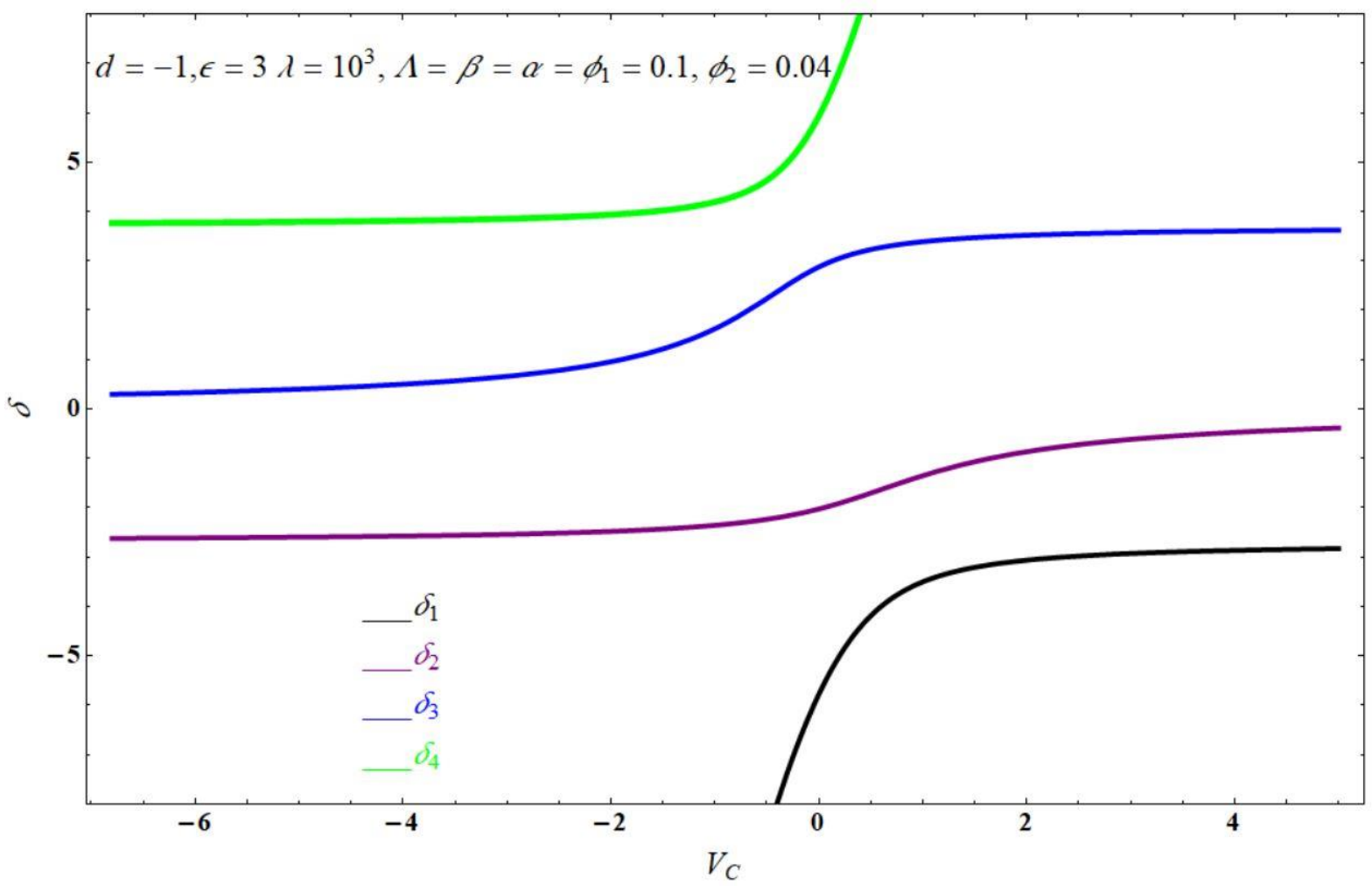

(b)

Fig.3: Effect of $\delta$ on $V_{C}$ for single value of $\varepsilon$ at (a) $d>0$ and (b) $d<0$. 


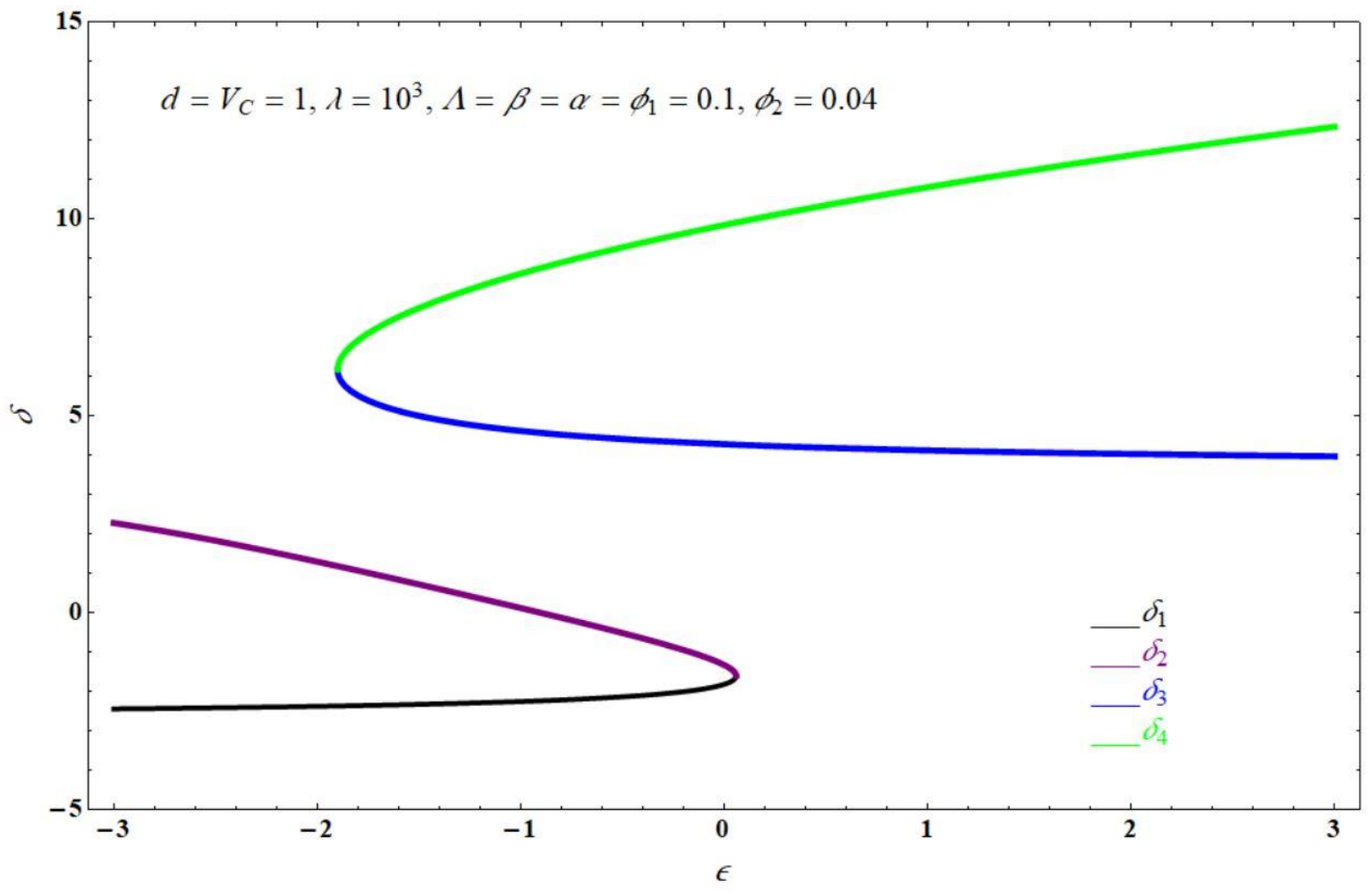

(a)

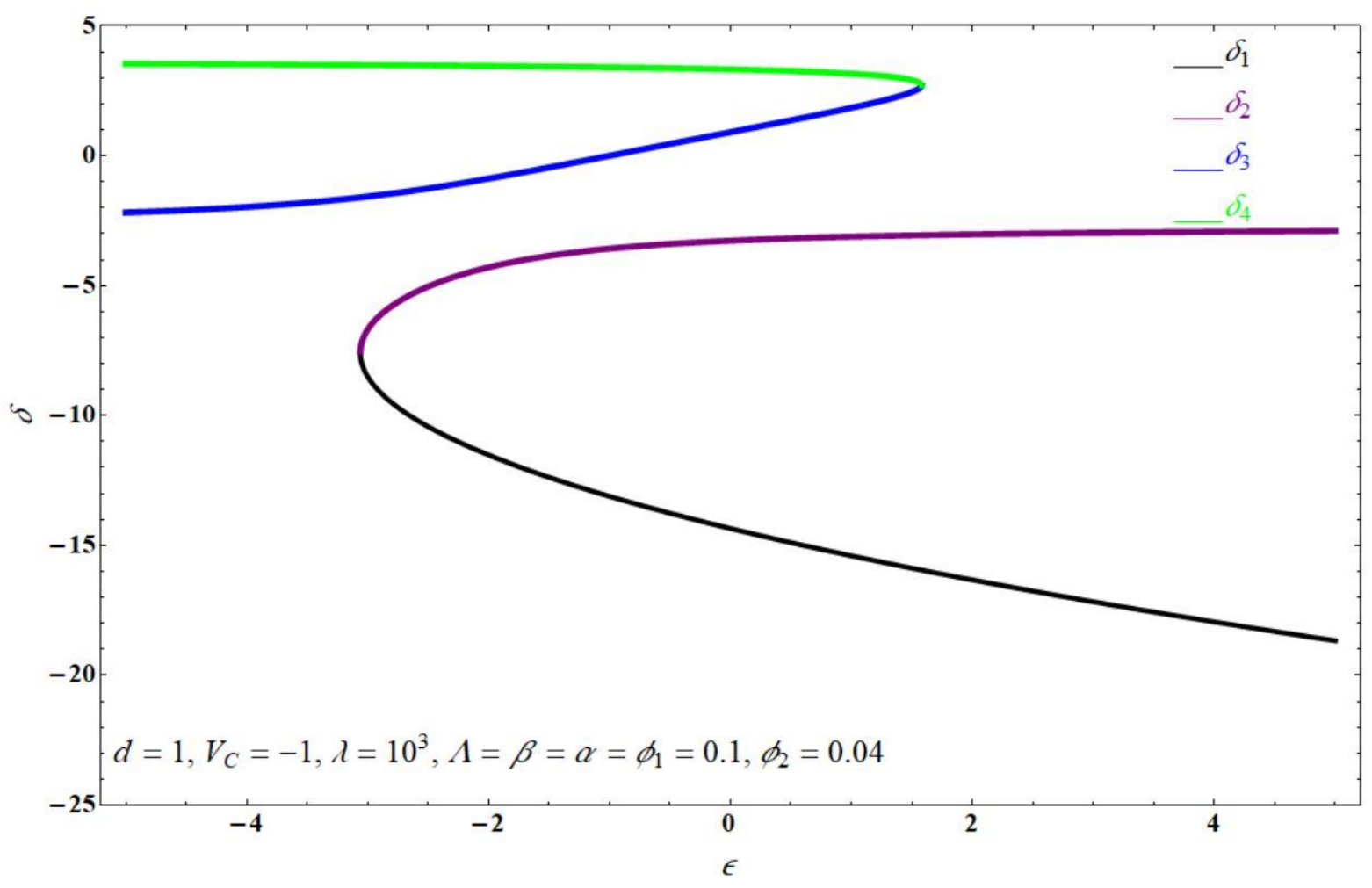

(b)

Fig.4: Effect of $\delta$ on $V_{C}$ for single value of $\varepsilon$ at (a) $d>0$ and (b) $d<0$. 


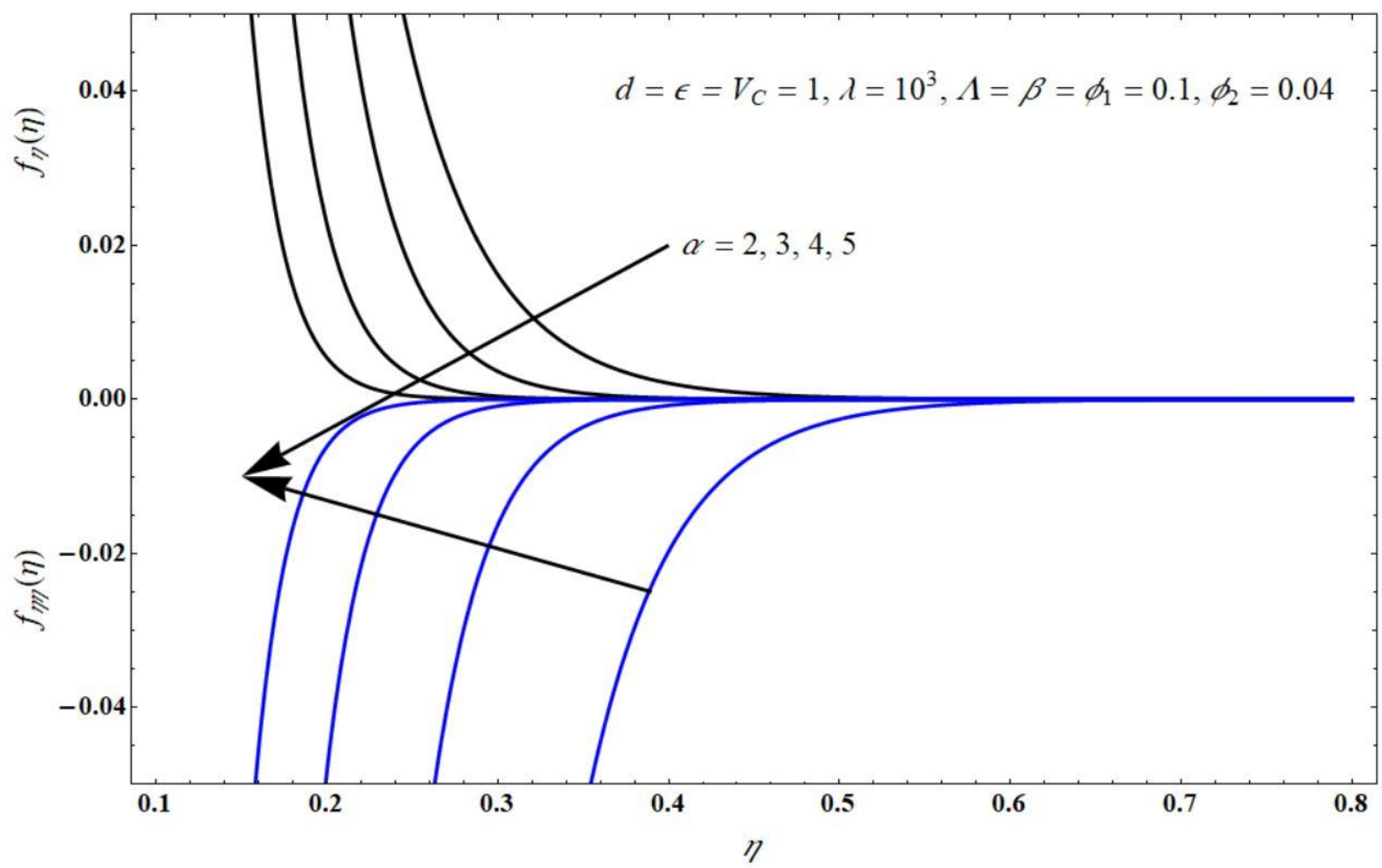

(a)

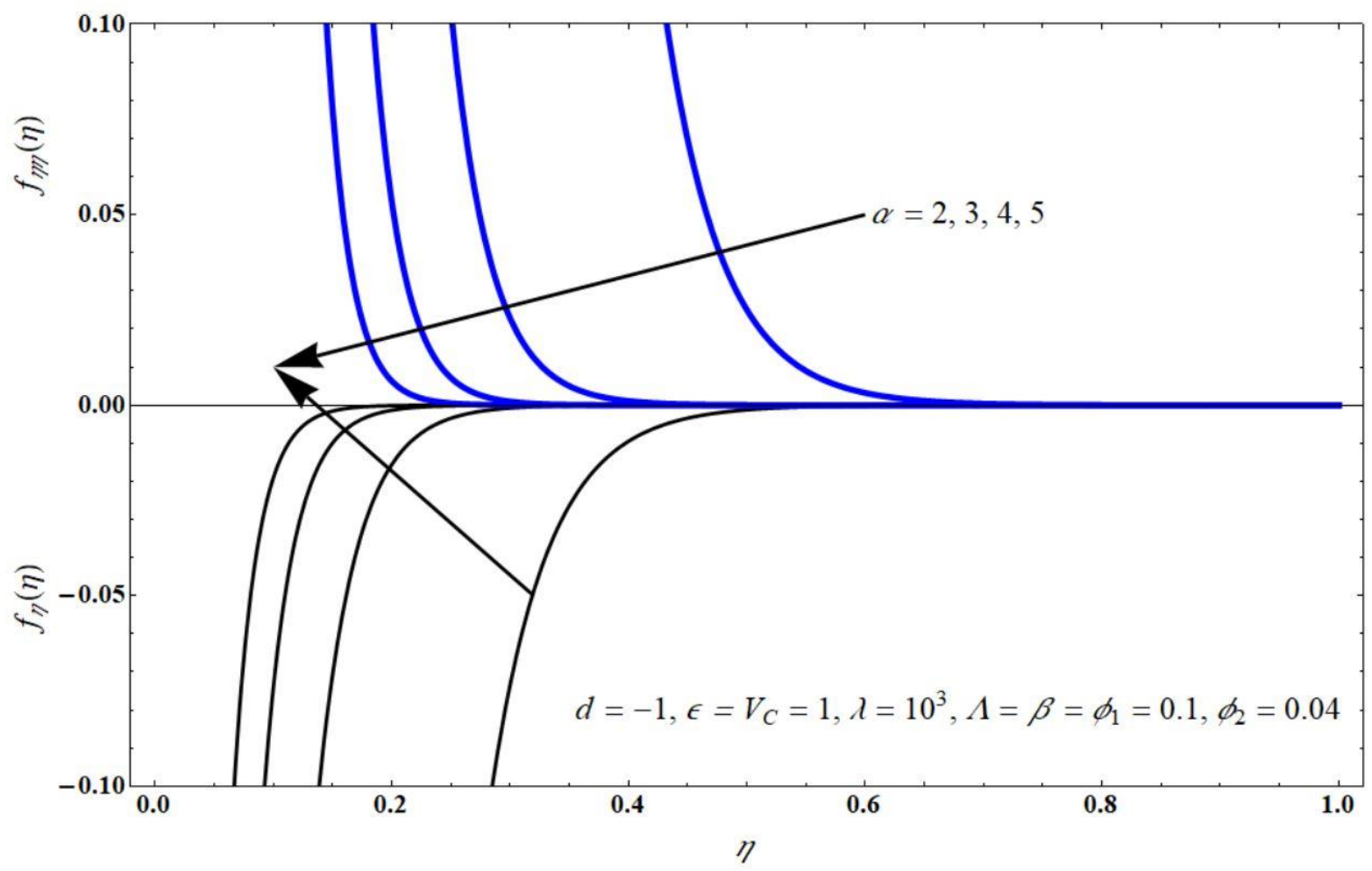

(b)

Fig.5: Impact of $f_{\eta}(\eta)$ and $f_{\eta \eta}(\eta)$ on $\eta$ for different choices of $\alpha$ at (a) $d>0$ and (b) $d<0$. 


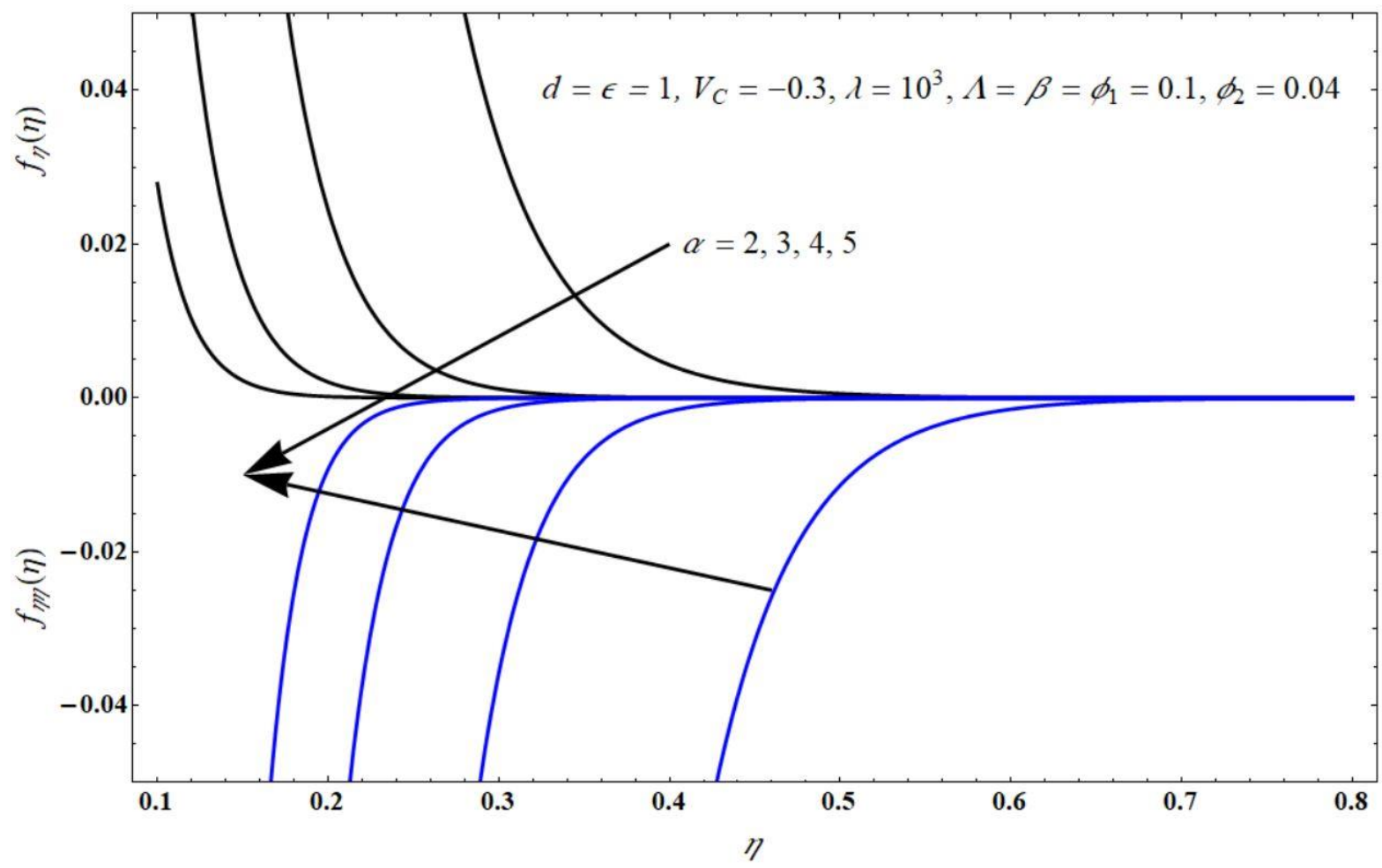

(a)

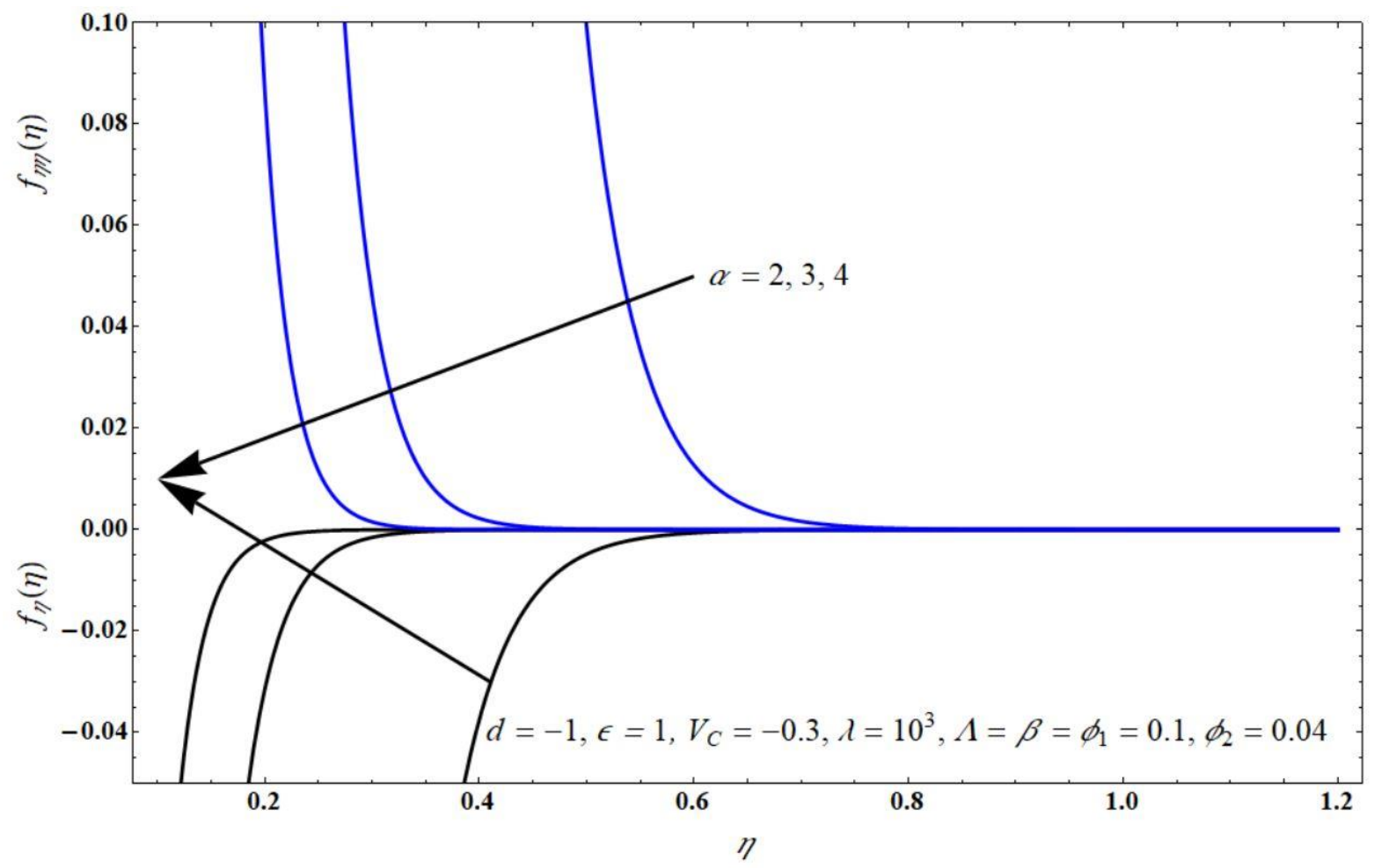

(b)

Fig.6: Impact of $f_{\eta}(\eta)$ and $f_{\eta \eta}(\eta)$ on $\eta$ for various choices of $\alpha$ at (a) $V_{C}>0$ and (b) $V_{C}<0$. 


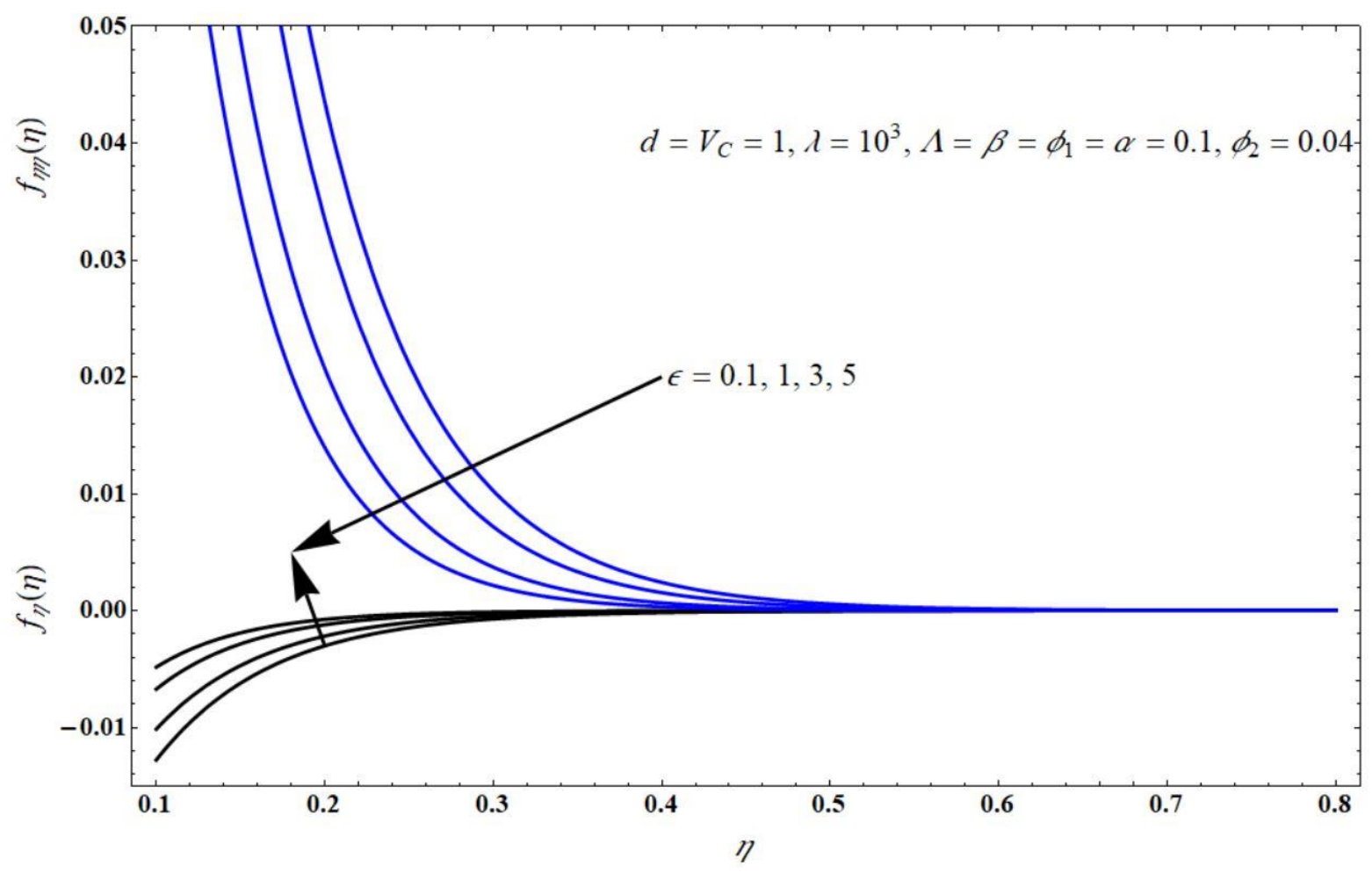

(a)

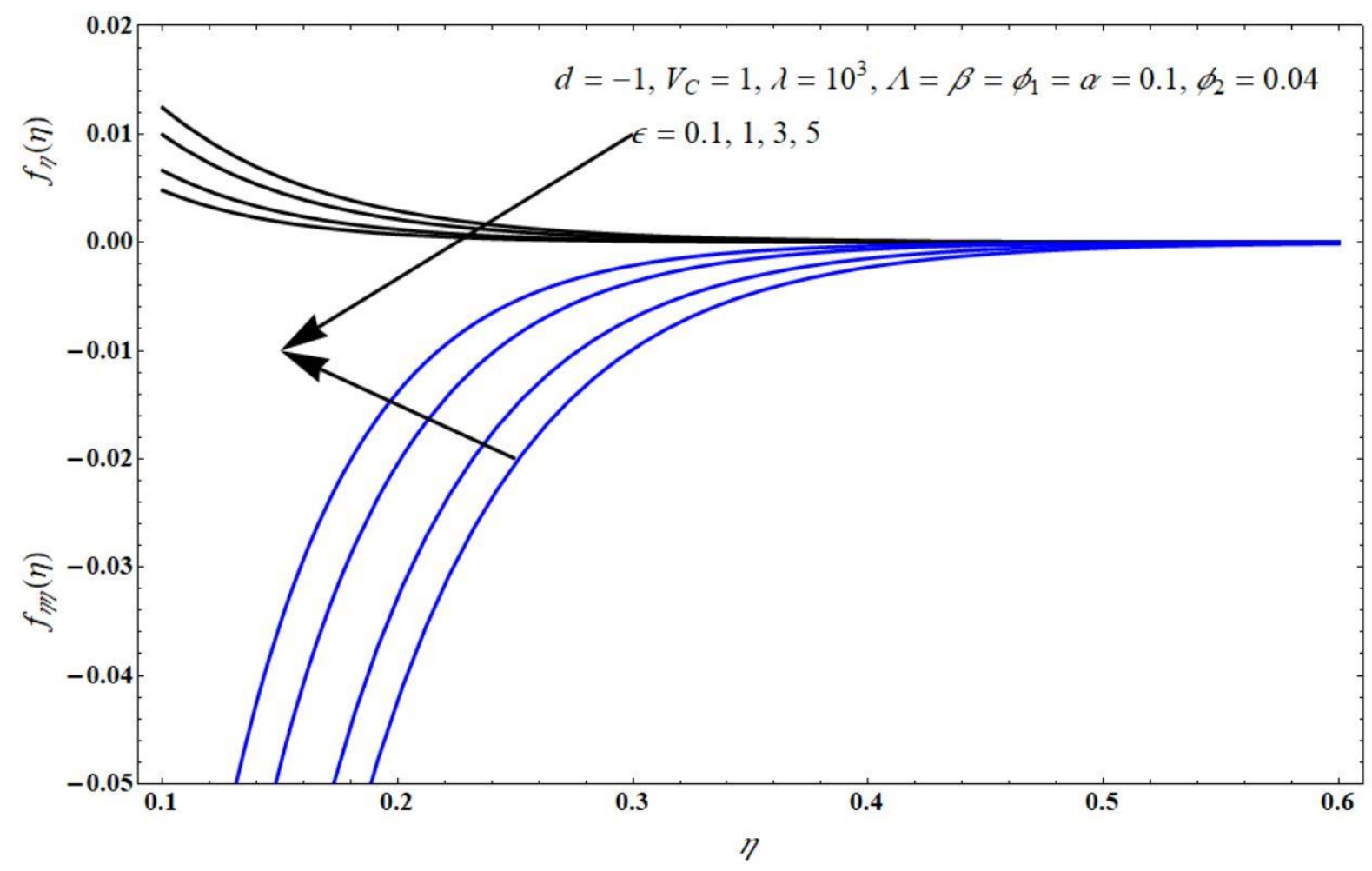

(b)

Fig.7: The impact of $f_{\eta}(\eta)$ and $f_{\eta \eta}(\eta)$ on $\eta$ for various choices of $\varepsilon$ at (a) $d>0$ and (b) $d<0$. 


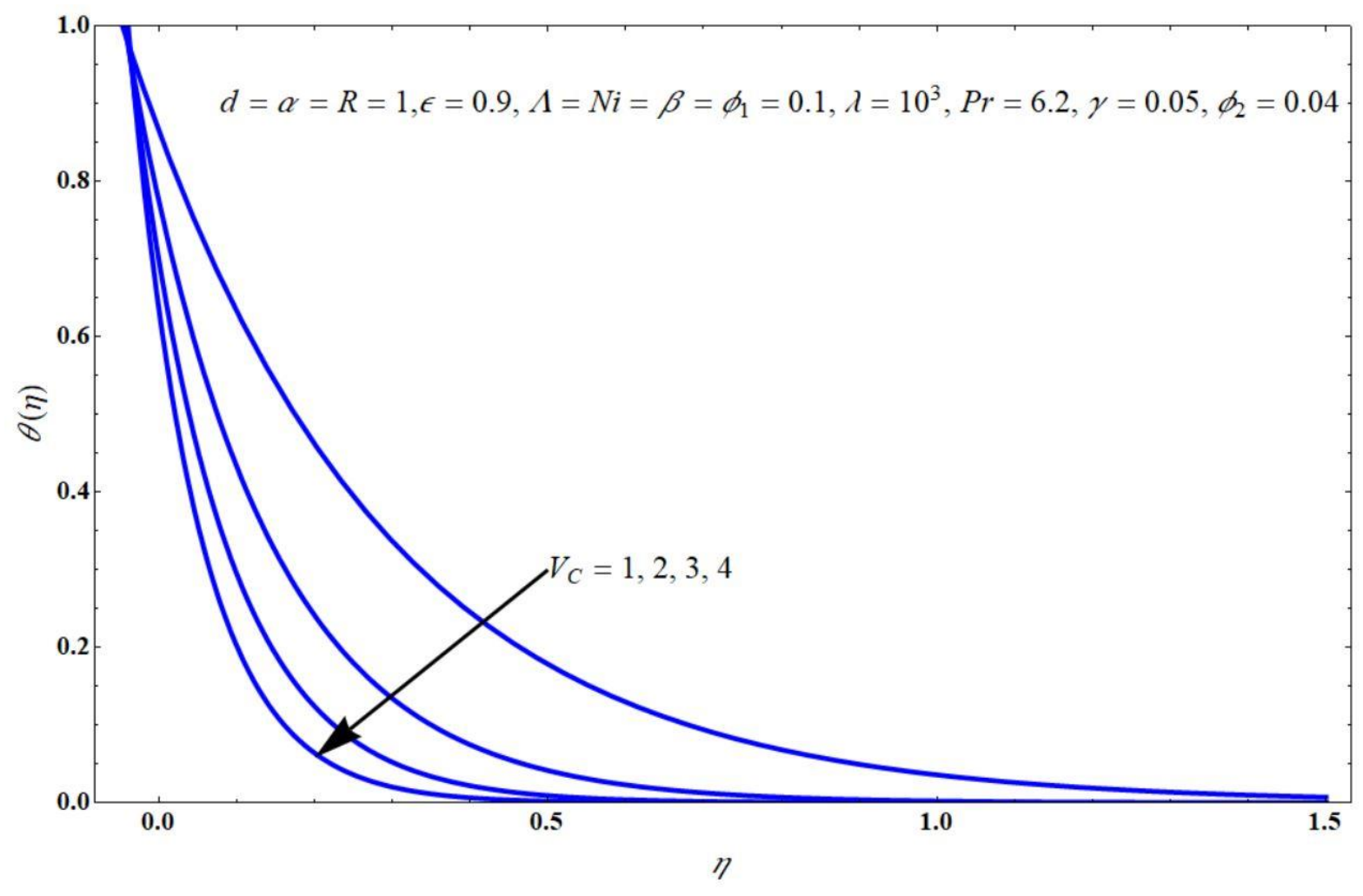

(a)

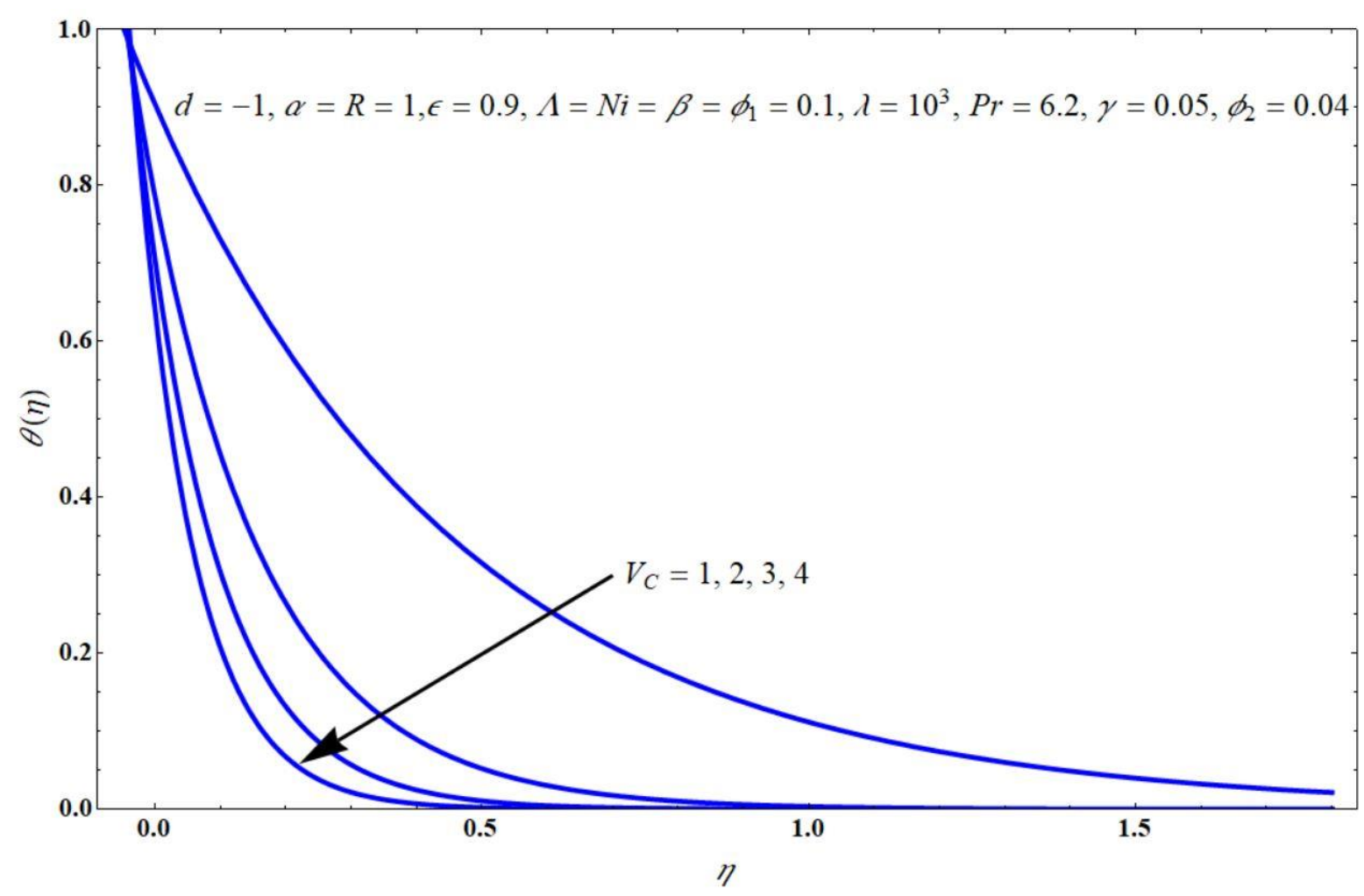

(b)

Fig.8: The impact of $\theta(\eta)$ on $\eta$ for various choices of $V_{C}$ at (a) $d>0$ and (b) $d<0$. 


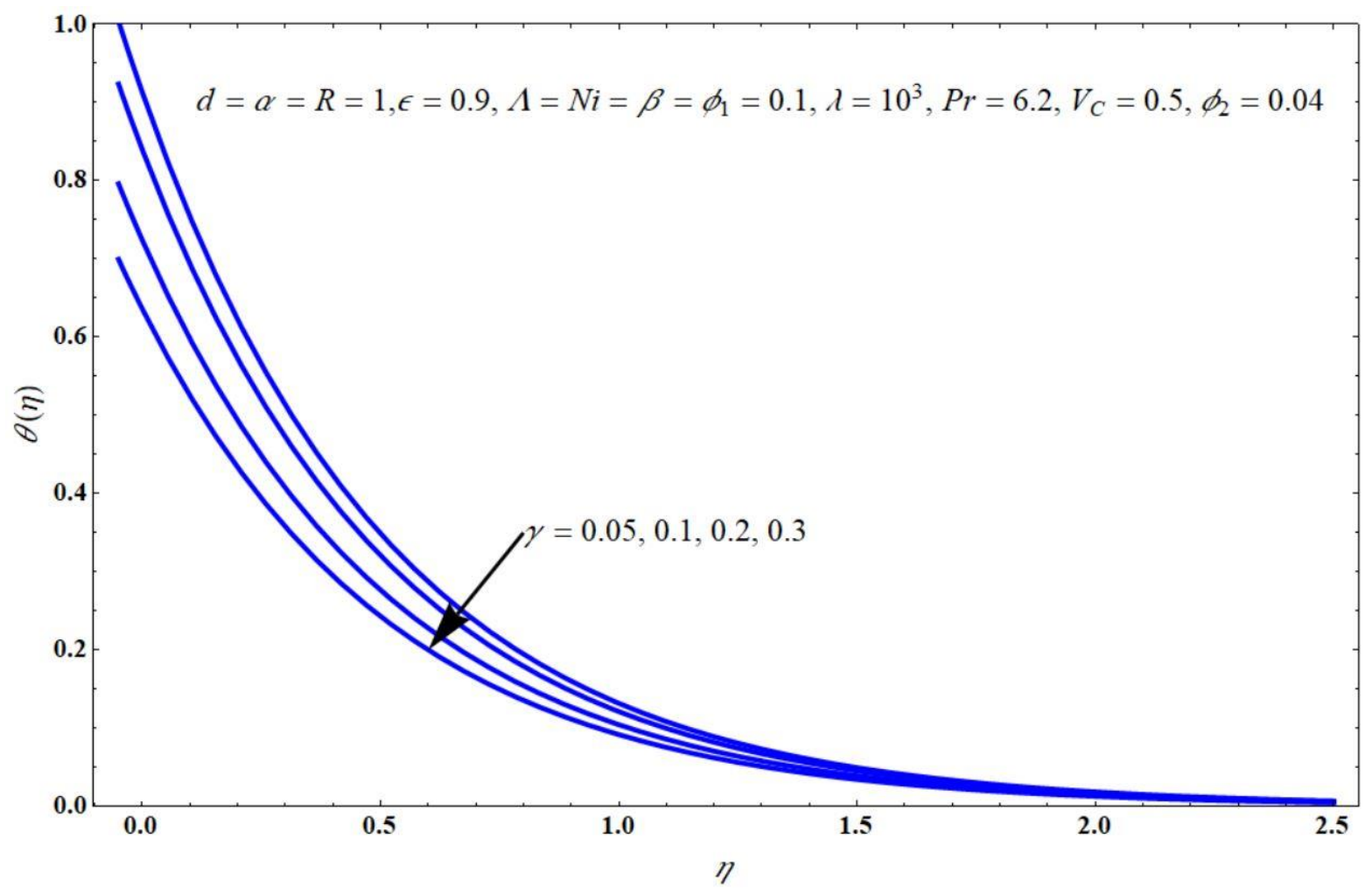

(a)

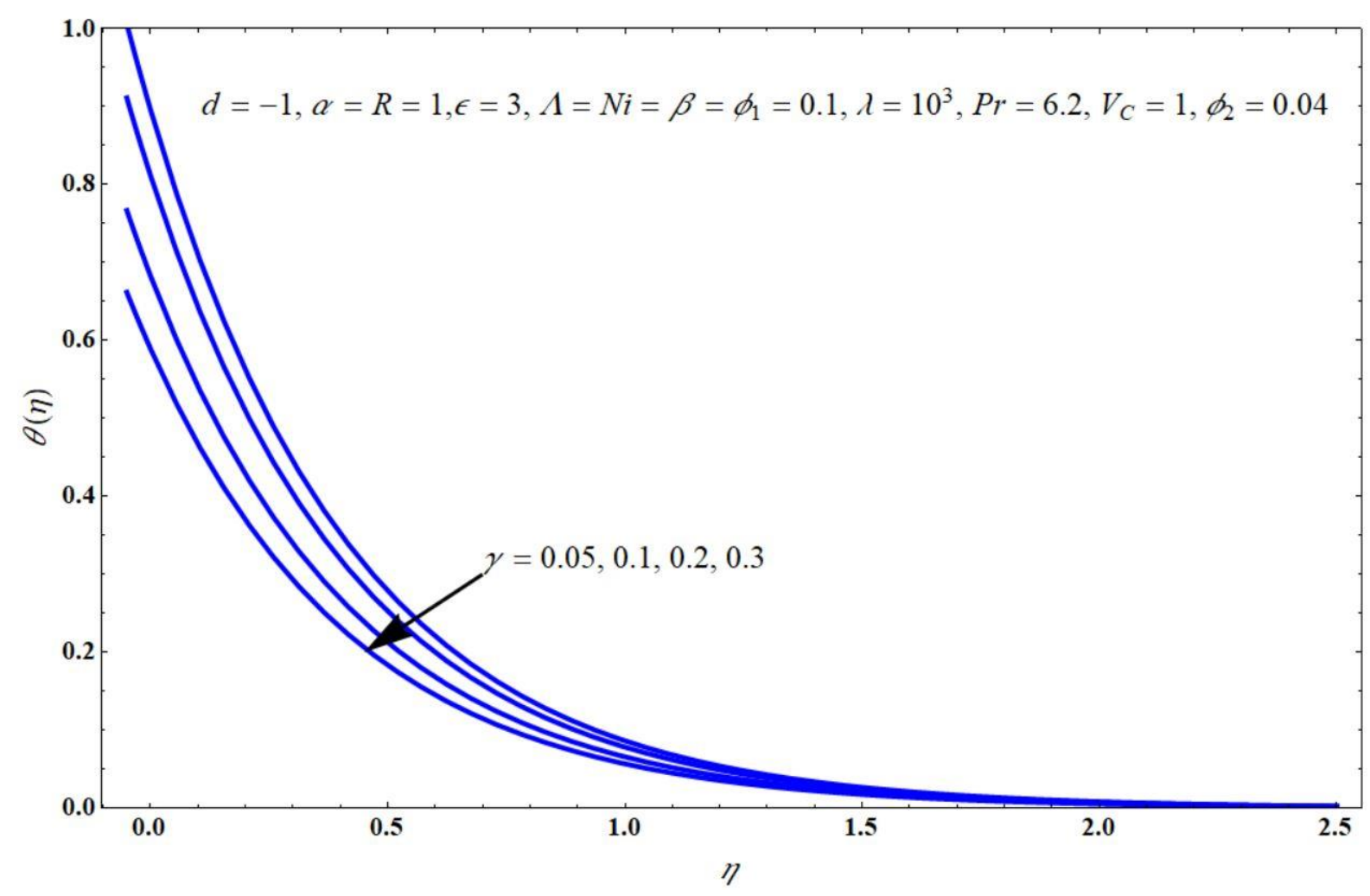

(b)

Fig.9: The impact of $\theta(\eta)$ on $\eta$ for various choices of $\gamma$ at (a) $d>0$ and (b) $d<0$. 


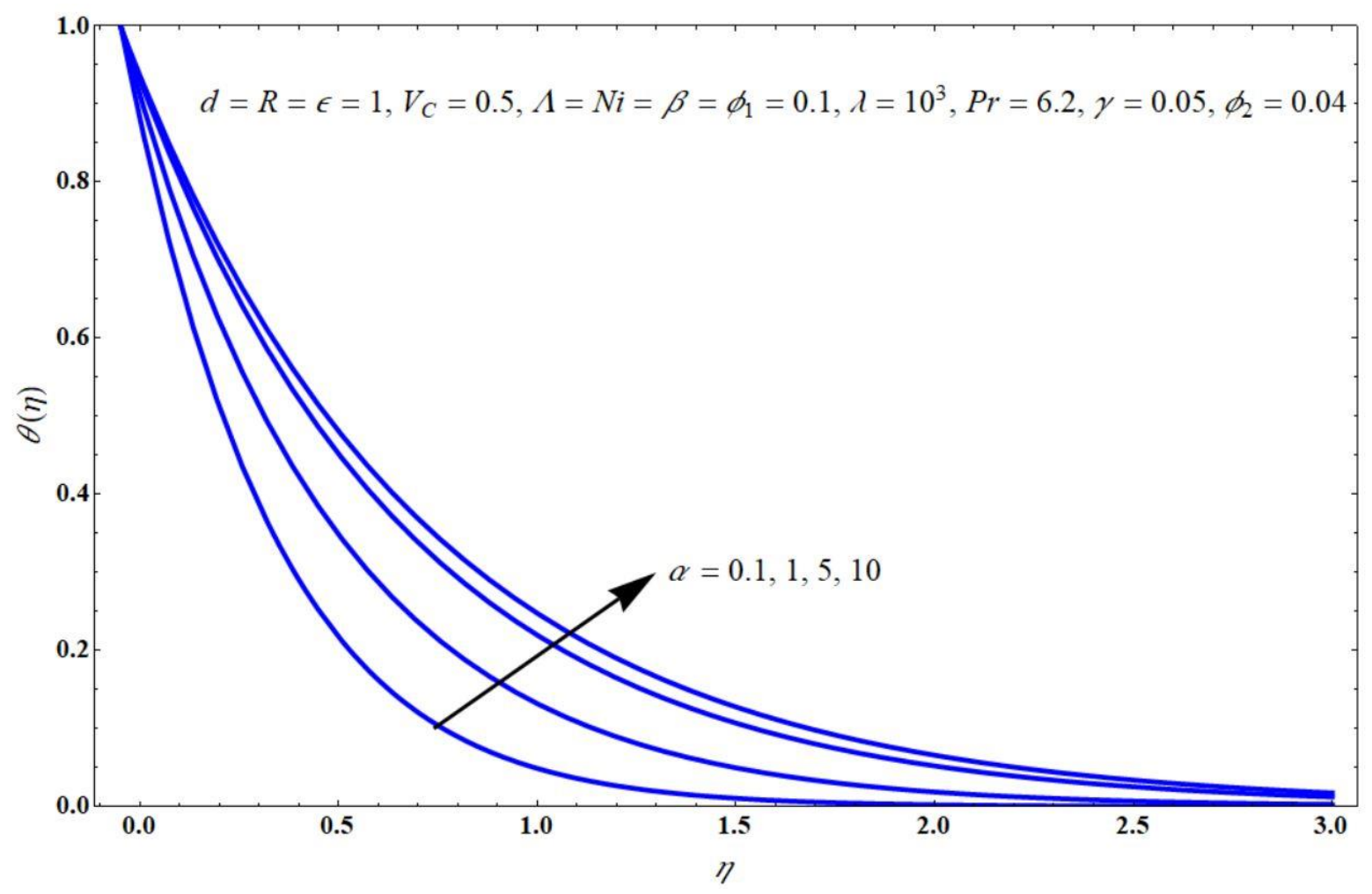

(a)

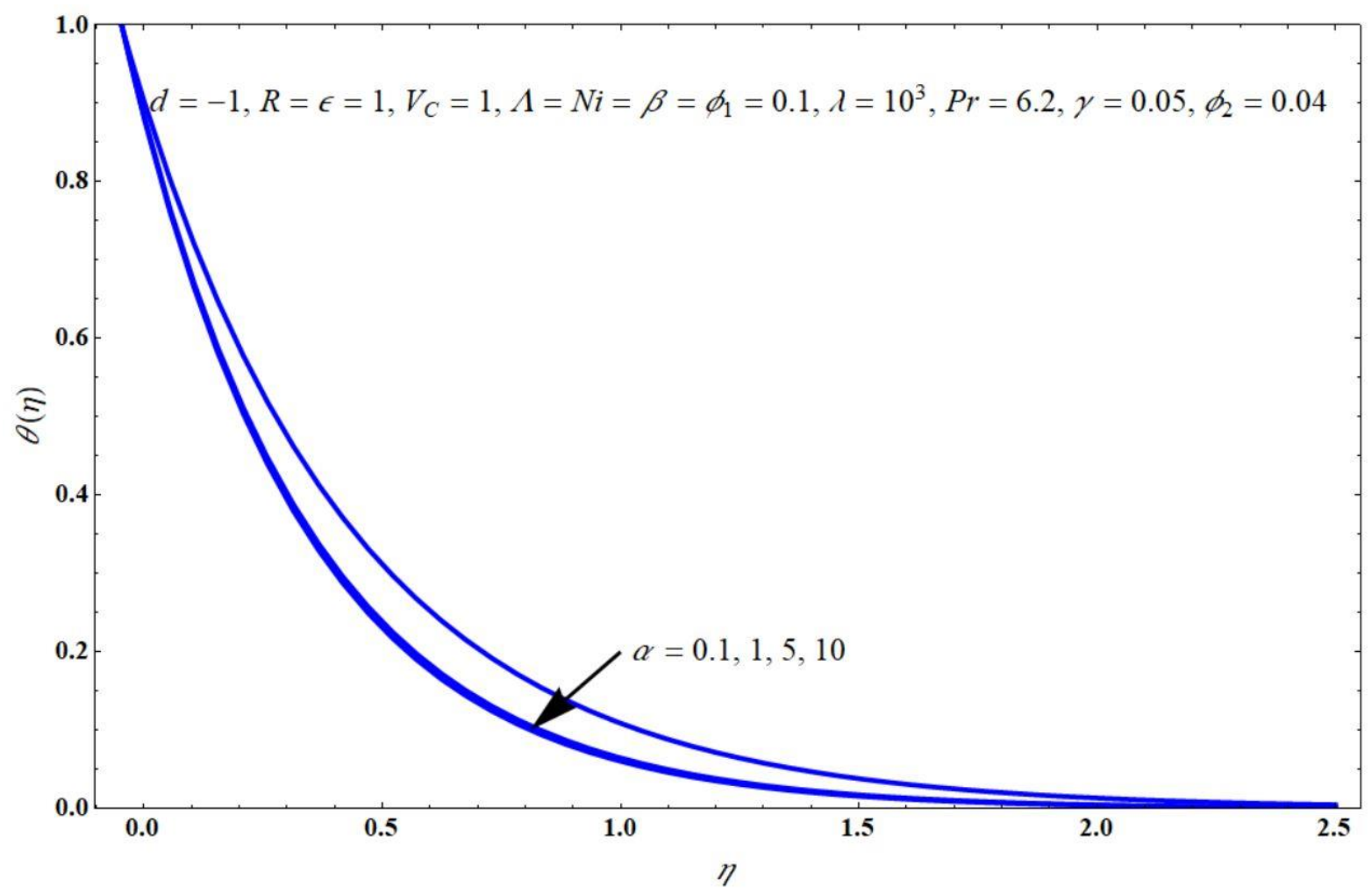

(b)

Fig.10: The impact of $\theta(\eta)$ on $\eta$ for various choices of $\alpha$ at (a) $d>0$ and (b) $d<0$. 


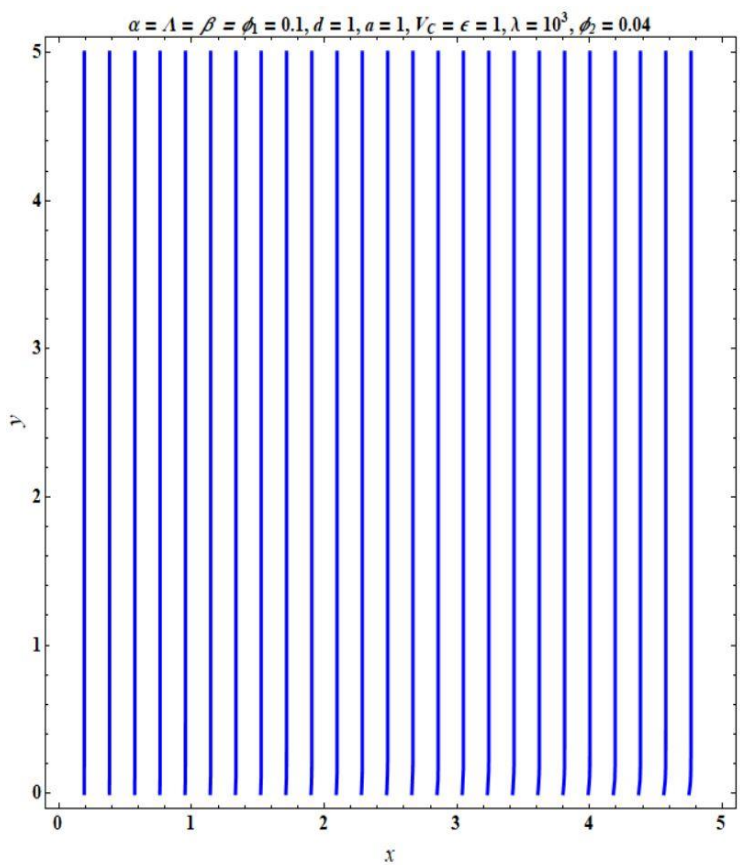

(a)

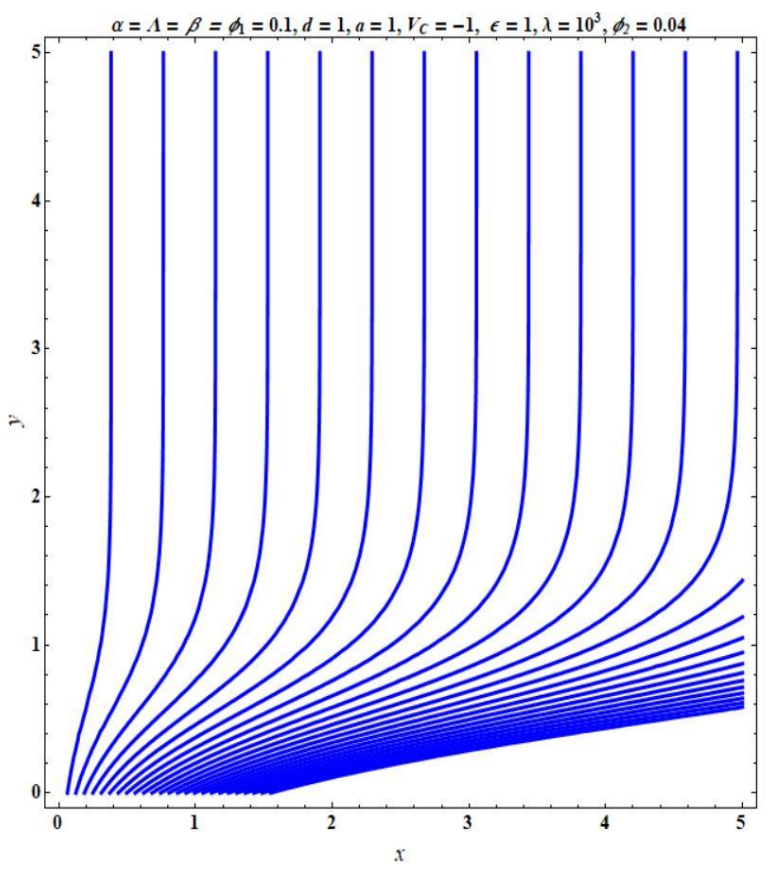

(b)

Fig.11: Pattern of streamline flows at (a) $V_{C}>0$ and (b) $V_{C}<0$.

\section{Concluding remarks}

A steady 2-D flow over a porous sheet with heat transfer is investigated in the current article.

The impact of Brinkmann number and mass transpiration is considered under momentum equation, also the thermal radiation is coming under energy equation. The PDEs of the given problem is mapped into ODEs with similarity variables. Then the momentum and energy equation is solved analytically to get the solution domain and energy equation in terms of energy equation. With the help of graphical scenario, we conclude the following results

- The graphs are plotted under four roots namely $\delta_{1} \delta_{2} \delta_{3}$ and $\delta_{4}$

- $\delta_{1}$ and $\delta_{2}$ values increases with increases of the values of $\varepsilon$ and $\delta_{3}$ and $\delta_{4}$ decreases with increases of the values of $\varepsilon$ for stretching case

- $\delta_{1}$ and $\delta_{3}$ values increases with increases of the values of $\varepsilon$ and $\delta_{2}$ and $\delta_{4}$ decreases with increases of the values of $\varepsilon$ for shrinking case 
- $f_{\eta}(\eta)$ is less for more values of $\alpha$. And $f_{\eta \eta}(\eta)$ more for more values of $\alpha$ for stretching case.

- $f_{\eta \eta}(\eta)$ less for more values of $\alpha$. And $f_{\eta}(\eta)$ is more for more values of $\alpha$ for shrinking sheet case.

- $\theta(\eta)$ less for more values of $V_{C}$ and $\gamma$ for both stretching and shrinking case

- $\theta(\eta)$ increases with increases of $\alpha$ for stretching case and $\theta(\eta)$ decreases with increases of $\alpha$ for shrinking case.

Data Availability Statement: The datasets used and analysed during the current study available from the corresponding author on reasonable request.

\section{References:}

1. S. Jana, A. Salehi-Khojin, W.H. Zhong, Enhancement of fluid thermal conductivity by the addition of single and hybrid nano-additives, Thermochimica acta 462(1-2) (2007) 45-55.

2. J.C. Maxwell, A Treatise on Electricity and Magnetism, Clarendon, Oxford, 1873.

3. S.U.S. Choi, J.A. Eastman, Enhancing thermal conductivity of fluids with nanoparticles, Am. Soc. Mech. Eng. Fluids Eng. Div. FED. 231 (1995) 99-105.

4. E.H. Aly, Existence of the multiple exact solutions for nanofluids flow over a stretching/shrinking sheet embedded in a porous medium at the presence of magnetic field with electrical conductivity and thermal radiation effects, Powder Tech. 301 (2016) 760781.

5. L.T. Benos, D.P. Nickolas, U.S. Mahabaleshwar, G. Lorenzini, I.E. Sarris. Thermal and flow investigation of MHD natural convection in a nanofluid-saturated porous enclosure: an asymptotic analysis. Jour. Therm. Analysis and Calorimetry. 143 (2021) 751-765.

6. U.S. Mahabaleshwar, A.B. Vishalakshi, M.N. Azese. The role of Brinkmann ratio on nonNewtonian fluid flow due to a porous shrinking/stretching sheet with heat transfer. European Jour. Mech./B fluids. 92 (2022) 153-165.

7. U.S. Mahabaleshwar, P.N. Vinay Kumar and M. Sheremet, "Magneto hydrodynamics flow of a nanofluid driven by a stretching/shrinking sheet with suction", Springer Plus. 5 (2016) 1-19. 
8. U.S. Mahabaleshwar, K.R. Nagaraju, P.N. Vinay Kumar, M.N. Nadagouda, R. Bennacer, M.A. Sheremet. Effects of Dufour and Soret mechanisms on MHD mixed convectiveradiative non-Newtonian liquid flow and heat transfer over a porous sheet. Therm. Sci. Eng. Pro. 16, (2020) 100459.

9. U.S Mahabaleshwar, K.R Nagaraju, P.N Vinay Kumar, M.N. Azese. Effect of radiation on thermosolutal Marangoni convection in a porous medium with chemical reaction and heat source/sink. Physics of fluid, 32 (2020) 113602.

10. U.S. Mahabaleshwar, G. Lorenzini. Combined effect of heat source/sink and stress work on MHD Newtonian fluid flow over a stretching porous sheet. Int. J. Heat and Calorimetry. 35, (2017), 330-335.

11. U.S. Mahabaleshwar, Ioannis E. Sarris \& Giulio Lorenzini Effect of radiation and Navier slip boundary of Walters' liquid B flow over a stretching sheet in a porous media, International Journal of Heat and Mass Transfer 127 (2018) 1327-1337.

12. A. Tamayol, K. Hooman, M. Bahrami, Thermal analysis of flow in a porous medium over a permeable stretching wall, Transp. Porous Media 85 (2010) 661-676.

13. T. Anusha, U.S. Mahabaleshwar, M. Hatami. Navier slip effect on the thermal-flow of Walter's liquid B flow due to porous stretching/shrinking with heat and mass transfer. Case Studies of thermal engineering, 28 (2021) 101691.

14. J. Sarkar, P. Ghosh, A. Adil, A review on hybrid nanofluids: recent research, development and applications, Renew. Sust. Energ. Rev. 43 (2015) 164-77.

15. T. Anusha, U.S. Mahabaleshwar, Y. Sheikhnejad. An MHD of nanofluid flow over a porous stretching/shrinking plate with mass transpiration and Brinkman ratio. Trans. Porous medium. (2021).

16. T. Anusha, Huang-Nan Huang, U.S. Mahabaleshwar. Two dimensional unsteady stagnation point flow of Casson hybrid nanofluid over a permeable flat surface and heat transfer analysis with radiation. Jour. Taiwan Inst. Chem. Eng. (2021).

17. K.N. Sneha, U.S. Mahabaleshwar, R. Bennacer, M EL Ganaoui. Darcy Brinkman equations for hybrid dusty nanofluid flow with heat transfer and mass transpiration. Computation. 9, (2021) 118.

18. U.S. Mahabaleshwar, A.B. Vishalakshi, H. I. Andersson. Hybrid nanofluid flow past a stretching/shrinking sheet with thermal radiation and mass transpiration. Chinese Jour. Phys. 75 (2022) 152-168.

19. U.S. Mahabaleshwar, T. Anusha, M. Hatami. The MHD Newtonian hybrid nanofluid flow and mass transfer analysis due to super-linear stretching sheet embedded in porous medium. Scientific reports, (2021)

20. W.K. Usafzai, E.H. Aly, A.S. Alshomrani, M.Z. Ullah. Multiple solutions for nanofluids flow and heat transfer in porous medium with velocity slip and temperature jump. Int. Commun. Heat and mass Transf. 131 (2020) 105831. 
21. U.S. Mahabaleshwar, K.N. Sneha, Huang-Nan-Huang. An effect of MHD and radiation on CNTS-water based nanofluid due to a stretching sheet in a Newtonian fluid. Case studies of thermal engineering. (2021) 101462.

22. U.S. Mahabaleshwar, T. Anusha, P.H. Sakanaka, S. Bhattacharyya. Impact of inclined Lorentz Force and Schmidt number on chemically reactive Newtonian fluid flow on a stretching surface when Stefan blowing and thermal radiation are significant. Arab. J. Sci. Eng. (2021).

23. A.B. Vishalakshi, U.S. Mahabaleshwar, I.E. Sarris. An MHD fluid flow over a porous stretching/ shrinking sheet with slips and Mass transpiration. Micromachines, 13 (2022) 116.

24. Aslani KE, Mahabaleshwar US, Jitender S, Sarries IE. Combined effect of radiation and inclined MHD flow of a micropolar fluid over a porous stretching/shrinking sheet with mass transpiration. Int. Jour. Appl. Comput. Math. 7, (2021) 1-21.

25. U.S. Mahabaleshwar. Stretching sheet and convective instability problems in Newtonian, micropolar and viscoelastic liquids. (2005) Ph. D. Thesis, Bangalore University, India.

26. L.J. Crane, Flow past a stretching plate, Z. Angew. Math. Phys. 21 (4) (1970) 645-647.

27. M. Miklavcic, C.Y. Wang, Viscous flow due to a shrinking sheet, Q. Appl. Math. 64 (2006) 283-290.

28. T. Fang, J. Zhang, Closed-form exact solutions of MHD viscous flow over a shrinking sheet, Commun. Nonlinear Sci. Numer. Simul. 14 (2009) 2853-2857.

Acknowledgements : This research received funding support from the NSRF via the Program Management Unit for Human Resources \& Institutional Development, Research and Innovation (grant number B05F640088).

Author contributions Conceptualization, U.S.M and T.B.; methodology, U.S.M, and T.B.; software, A.B.V.; validation, T.B.; formal analysis, U.S.M. and A.B.V.; investigation, U.S.M and T.B.; writing - original draft preparation, U.S.M and T.B.; writing - review and editing, U.S.M and T.B.; project administration, T.B.; funding acquisition, U.S.M and T.B. All authors have read and agreed to the published version of the manuscript.

Conflicts of Interest: The authors declare that they have no conflicts of interest to report regarding the present study. 